Florida International University FIU Digital Commons

FIU Electronic Theses and Dissertations

University Graduate School

$1-26-2012$

\title{
Hurricane Loss Modeling and Extreme Quantile Estimation
}

Fan Yang

Florida International University, fyang001@fiu.edu

DOI: $10.25148 /$ etd.FI12041106

Follow this and additional works at: https://digitalcommons.fiu.edu/etd

\section{Recommended Citation}

Yang, Fan, "Hurricane Loss Modeling and Extreme Quantile Estimation" (2012). FIU Electronic Theses and Dissertations. 557.

https://digitalcommons.fiu.edu/etd/557

This work is brought to you for free and open access by the University Graduate School at FIU Digital Commons. It has been accepted for inclusion in FIU Electronic Theses and Dissertations by an authorized administrator of FIU Digital Commons. For more information, please contact dcc@fiu.edu. 


\section{FLORIDA INTERNATIONAL UNIVERSITY \\ Miami, Florida}

HURRICANE LOSS MODELING AND EXTREME QUANTILE ESTIMATION

A thesis submitted in partial fulfillment of the

requirements for the degree of

MASTER OF SCIENCE

in

STATISTICS

by

Fan Yang

2012 


\section{To: Dean Kenneth Furton}

College of Arts and Sciences

This thesis, written by Fan Yang, and entitled Hurricane Loss Modeling and Extreme Quantile Estimation, having been approved in respect to style and intellectual content, is referred to you for judgment.

We have read this thesis and recommend that it be approved.

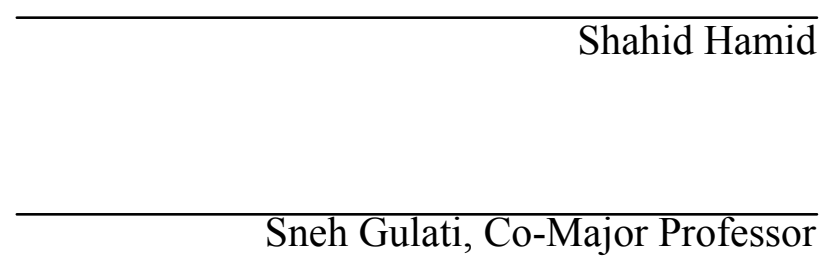

B.M. Golam Kibria, Co-Major Professor

Date of Defense: January 26, 2012

The thesis of Fan Yang is approved.

Dean Kenneth Furton

College of Arts and Sciences

Dean Lakshmi N. Reddi

University Graduate School

Florida International University, 2012 


\section{DEDICATION}

I dedicate this thesis to my mentor Daisaku Ikeda. He has taught me the attitude of learning, the meaning of education and inner strength. Without his guidance, encouragement and love, the completion of this work would not have been possible and I could not become the person I am today. 


\section{ACKNOWLEDGMENTS}

I offer my sincere gratitude to Prof. Sneh Gulati and Dr. B.M. Golam Kibria who have supported me throughout my thesis with their patience and knowledge whilst allowing me the room to work in my own way. I would like to thank Prof. Shahid Hamid for his helpful suggestions to improve my thesis.

I thank professors and classmates of Department of Statistics for great learning experience at FIU. Finally I want to express my sincere appreciation to my friend Yoshiko Yamada and so many others in SGI, my family in Miami, for their strong support and belief in me. 


\begin{abstract}
OF THE THESIS
HURRICANE LOSS MODELING AND EXTREME QUANTILE ESTIMATION
\end{abstract}

by

Fan Yang

Florida International University, 2012

Miami, Florida

Professor B. M. Golam Kibria, Co-Major Professor

Professor Sneh Gulati, Co-Major Professor

This thesis reviewed various heavy tailed distributions and Extreme Value

Theory (EVT) to estimate the catastrophic losses simulated from Florida Public

Hurricane Loss Projection Model (FPHLPM). We have compared risk measures such as Probable Maximum Loss (PML) and Tail Value at Risk (TVaR) of the selected distributions with empirical estimation to capture the characteristics of the loss data as well as its tail distribution. Generalized Pareto Distribution (GPD) is the main focus for modeling the tail losses in this application. We found that the hurricane loss data generated from FPHLPM were consistent with historical losses and were not as heavy as expected. The tail of the stochastic annual maximum losses can be explained by an exponential distribution.

This thesis also touched on the philosophical implication of small probability, high impact events such as Black Swan and discussed the limitations of quantifying catastrophic losses for future inference using statistical methods. 


\section{TABLE OF CONTENTS}

CHAPTER

PAGE

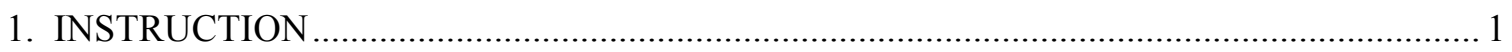

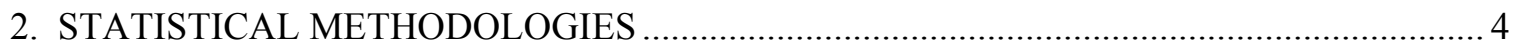

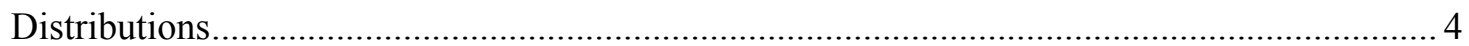

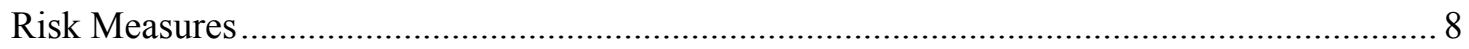

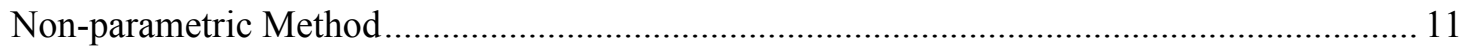

3. PARAMETER ESTIMATION ………………………………………………………..... 13

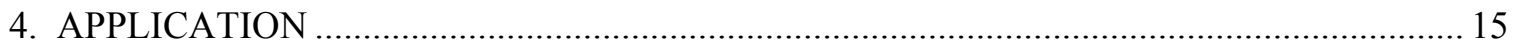

Losses Generated from a Stochastic Hurricane Set ........................................................... 15

Losses Generated from Historical Landfalls......................................................................... 27

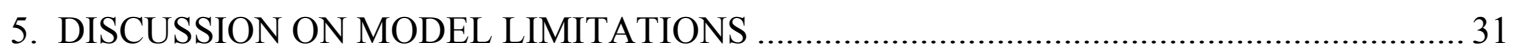

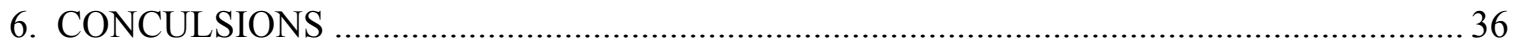

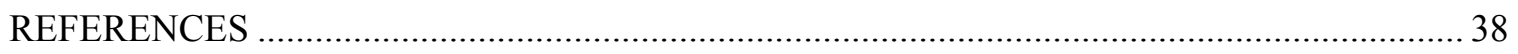

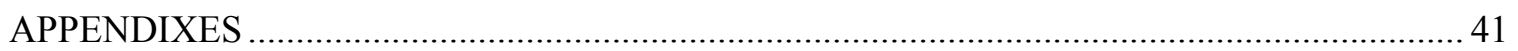




\section{LIST OF TABLES}

TABLES

PAGE

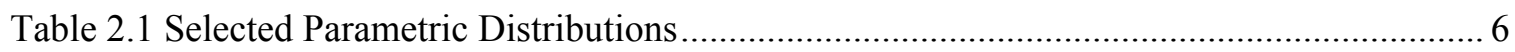

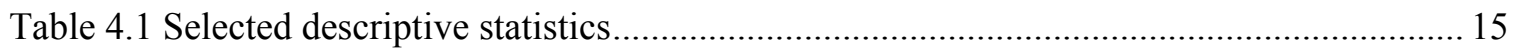

Table 4.2 Shape parameter comparison, per occ data .............................................................. 19

Table 4.3 Shape parameter comparison, per agg …............................................................... 20

Table 4.4 Risk measures for different thresholds, per agg...................................................... 21

Table 4.5 MLE and MoM comparison of extreme risk measures.............................................. 21

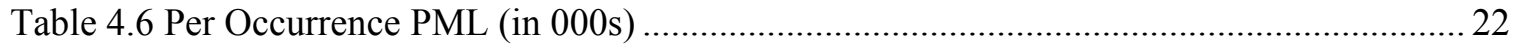

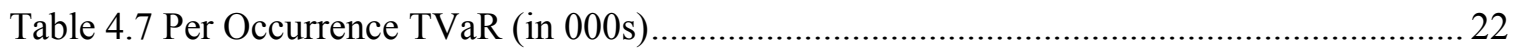

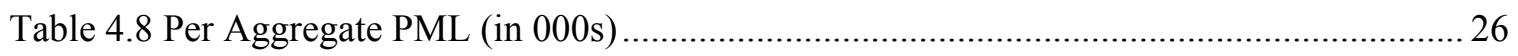

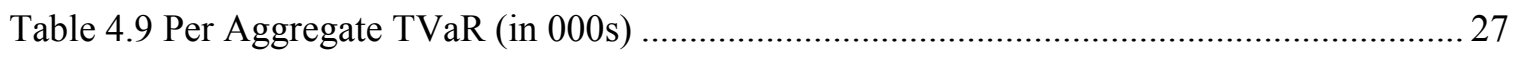

Table 4.10 Descriptive statistics for historical data and per agg data ........................................ 28

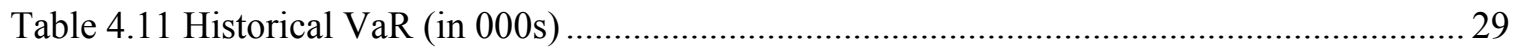

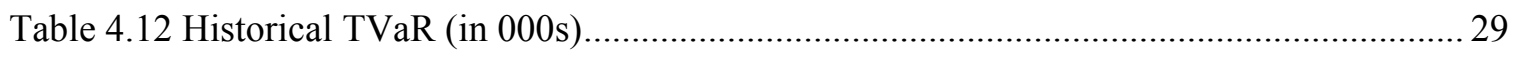

Table 5.1 Empirical PML estimation with 95 \% confidence limits ............................................ 33

Table 5.2 Risk measure comparison for per aggregate data using GPD ..................................... 33 


\section{LIST OF FIGURES}

FIGURE

PAGE

Figure 4.1 Histograms for annual maximum losses and annual aggregate loss ...............15

Figure 4.2 Skewness-Kurtosis plot for per occ data with 100 bootstrap samples ............16

Figure 4.3 Mean Excess Plot for per occ data with 95\% confidence limits ....................17

Figure 4.4 Shape parameter variability plot for per occ data......................................18

Figure 4.5 Shape estimates with 95\% confidence limits, per occ................................19

Figure 4.6 Shape estimates with 95\% confidence limits, per agg ...............................20

Figure 4.7 CDF comparison for modeling annual maximum losses ...........................23

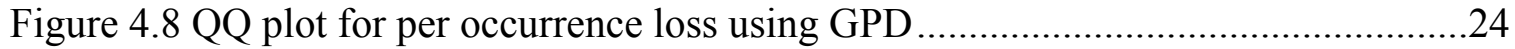

Figure $4.995 \%$ confidence intervals for $99^{\text {th }}$ percentile with varying threshold, per occ .25

Figure 4.10 QQ plot for per aggregate loss using GPD ..........................................26

Figure 4.11 Histogram of losses generated by historical land falling and by-passing

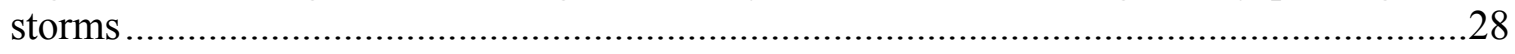

Figure 4.12 Diagnostic plots for GPD modeling historical losses...............................30

Figure 4.13 QQ plot for historical losses and stochastic losses .................................30 


\section{Chapter 1}

\section{INTRODUCTION}

Over the last few decades, the study of extreme values has gained importance for prediction and risk management purposes in various fields. In finance and insurance, extreme losses are a primary focus because of their impact on firms' solvency and sustainability. Insurers are particularly concerned with a sudden surge of large claims caused by natural disasters, also referred to as catastrophic (cat) events such as a major earthquake or a hurricane, resulting in loss of life and serious damage to buildings and their contents. Potential risks inherent in the low frequency but high severity event, have necessitated the development of proper risk quantification through cat modeling.

Cat model is a mathematical tool that quantifies risks from cat events including hurricanes, earthquakes, and terrorism in terms of loss severity and frequency. Commercial modeling companies such as AIR, RMS and EQECAT have developed such models that insurers use to estimate and evaluate the potential cat risks based on the business exposure. Many companies turn to these models for various operational and strategic decision making including underwriting, pricing, reinsurance purchase and capital allocation. Consequently, in the property and casualty insurance business, it has become an industry standard to calculate and disclose extreme measures including VaR (Value-at-Risk) and TVaR (Tail Value-at-Risk) for quantifying losses.

Given the complexity of modeling cat event losses, a good estimation model relies on interdisciplinary collaboration of scientists, actuaries and software-developers. The computer-based models utilize historical records as well as current scientific research on 
natural hazards to assess the cat risks. With the help of advanced computer power and Geographic Information System (GIS) software, nowadays the models can map losses at the street level (Gilli and Evis, 2003).

The International Hurricane Research Center (IHRC) is a multidisciplinary hurricane research facility within the State of Florida University System. The Florida Public Hurricane Loss Model (FPHLM) developed at IHRC uses an Oracle database and is available to the public. The model consists of three modules: meteorology, engineering and finance/insurance. Stochastic event catalogs produce probabilities and intensities of wind events that produce losses. Damage matrices are established by engineers to assess the damage to properties caused by these events. In the insurance module, losses are calculated for a variety of policy features. The model generates hurricane losses through a 54,000 year-run stochastic hurricane set. For more information on the model we refer the readers to Hamid et al. $(2008,2010)$ and references therein. My thesis compares the simulated losses with those produced by the modeling of historical landfalls for Probable Maximum Loss (PML) calculations.

PML statistically corresponds to a high quantile of the loss distribution and is equivalent to VaR in the financial industry. There are three types of PML: per event (losses incurred in one cat event), per occurrence (the largest event loss within a year) or per aggregate (the total annual loss). The latter two are commonly used. Per occurrence PML gives various quantiles for possible maximum losses in a year, while per aggregate PML provides quantiles for annual aggregate losses. The FPHLM has traditionally used a nonparametric method for PML estimation, whereas this thesis examines the parametric 
method. Both assume that the simulated losses are a reasonable representation of the population of hurricane losses.

The probabilistic framework for modeling cat losses builds on heavy tail distributions as well as Extreme Value Theory (EVT). Generalized Pareto Distribution (GPD) is the main focus for modeling the tail losses in this research. Extreme losses from hurricanes make classical statistical analysis based on normality assumptions inappropriate. One challenge of extreme value modeling is to balance the bias and variance. The asymptotic result, which is a large sample property, poses applicability constraints and the small sample issue creates problems when it comes to statistical inference. For example, GPD relies on limit theorems; to be able to correctly use the limit theory and make an inference, a certain threshold must be surpassed. Yet, in general there is less data above this cutoff point, making the estimators more variable. Lowering the threshold would provide more data and reduce the variance but also inevitably induce larger bias. However, this was not a major concern in my study since the simulated losses from the cat model contained 54,000 data points.

For the parametric method, the data was fitted to several distributions commonly used by practitioners in the insurance industry to calculate the quantiles. The obtained results were compared with those from the empirical method and also with historical losses for model validation. The organization of the thesis is as follows: Chapter 2 deals with the theoretical foundations of extreme value analysis and detailed mathematical descriptions can be found in the Appendix. Chapter 3 discusses parameter estimation and Chapter 4 discloses findings from the application of FPHLM data. Model limitations are discussed in Chapter 5. Finally some conclusions are presented in Chapter 6. 


\section{Chapter 2}

\section{STATISTICAL METHODOLOGY}

\subsection{Distributions}

Because the historical catastrophic data is limited and extreme quantile levels are required, finding an appropriate probabilistic model for hurricane losses is a challenge. The FPHLM solves the small sample problem by projecting the available historical data into a larger stochastic set of a long time horizon to conduct the risk analysis. The model is updated biannually to use current exposure to represent the latest population, building codes and replacement costs and incorporate other improvements as necessary.

The losses generated from FPHLM were presumed to be highly right skewed, requiring the usage of heavy-tailed distributions. The candidate models can be categorized into two groups, non-GPD for modeling the entire loss data and GPD for the exceedances above a chosen threshold.

\subsubsection{Non-GPD — Distribution for the Whole Data Range}

Quantification of risk is distribution-based and cat losses can be modeled by a heavytailed distribution in which the tail decays as a power function: $\mathrm{p}(\mathrm{X}>\mathrm{x}) \quad \mathrm{x}^{-\alpha 1}$ (Resnick, 2007). The tail behavior of the underlying model is essential in studying high quantile estimation. The quality of high quantile estimation largely depends on the second order behavior of the underlying slowly varying function $\mathrm{L}$ of the tail structure (Degen and Embrechts, 2007). Concepts of slowly varying function and regular variation which provide regularity conditions for this mathematical structure can be found in Appendix A.

\footnotetext{
${ }^{1} \mathrm{f}(\mathrm{x}) \quad \mathrm{g}(\mathrm{x})$ stands for $\lim _{x \rightarrow \infty} \frac{f(x)}{g(x)}=1$
} 
Various two-parameter heavy-tailed distributions such as Pareto, Lognormal and Loglogistic can be applied to the heavy tailed data. Relatively lighter tailed distributions ranging from Exponential, Weibull to Gamma provide nice comparisons to the above heavier-tailed distributions. We refer the reader to Kreps (2000) and Klugman et al. (2008) for a comprehensive summary of distributions commonly used to model insurance losses.

A four-parameter general class of distributions, Transform Beta (TB), which is also referred as Generalized Beta Distribution of Second Kind (GB2), was considered. The benefit of using general class distributions despite their complexity is the fact that they could be used to tentatively find out if the distributions under the same family would be a good fit (Dutta and Perry, 2006). Furthermore, a general distribution helps to reduce model risks from incorrectly specifying a particular distribution. Many heavy-tailed distributions can be generated from TB by specifying some parameters. Appendix B provides an illustration. Table 2.1 gives the distributions under consideration in this study. 
Table 2.1 Selected parametric distributions ${ }^{2}$

\begin{tabular}{|c|c|}
\hline Distribution & Density Function $f(x)$ \\
\hline Exponential & $\frac{1}{\theta} e^{-x / \theta} ; x>0$ and $\theta>0$ \\
\hline Gamma & $\frac{\left(x / \theta^{\alpha} e^{-x / \theta}\right.}{x \Gamma(\alpha)} ; x>0, \alpha>0$ and $\theta>0$ \\
\hline Lognormal & $\begin{array}{c}\frac{1}{x \sigma \sqrt{2 \pi}} e\left(-z^{2} / 2\right), z=\frac{\ln x-\mu}{\sigma} \\
x>0, \mu \in R \text { and } \sigma^{2}>0\end{array}$ \\
\hline Pareto & $\frac{\alpha \theta^{\alpha}}{(x+\theta)^{\alpha+1}} ; x>0, \alpha>0$ and $\theta>0$ \\
\hline Loglogistic & $\frac{\gamma(x / \theta)^{\gamma}}{x\left[1+(x / \theta)^{\gamma}\right]^{2}} x>0, \alpha>0$ and $\gamma>0$ \\
\hline Transform Beta & $\begin{array}{c}\frac{\gamma(x / \theta)^{\gamma \tau}}{B(\alpha, \tau) x\left[1+(x / \theta)^{\gamma}\right]^{\alpha+\tau}} B(\alpha, \tau)=\frac{\Gamma(\alpha) \Gamma(\tau)}{\Gamma(\alpha+\tau)} \\
x>0, \alpha>0, \theta>0, \gamma>0 \text { and } \tau>0\end{array}$ \\
\hline
\end{tabular}

\subsubsection{GEV and GPD - Distribution of the Tail}

2

Location-scale transformation: if $X$ has the $\mathrm{df} F$, then $\mu+\sigma X$ has the $\mathrm{df} \mathrm{F}_{\mu, \sigma}(\mathrm{x})=\mathrm{F}((\mathrm{x}-\mu) / \sigma)$. E.g. for Loglogistic distribution above, if there is no scaling $(\theta=1)$ and shift the loss to the right by $\mathrm{u}$, the density function will become $\frac{\gamma(x-u)^{\gamma-1}}{\left[1+(x-u)^{\gamma}\right]^{2}}$ 
The (right) tail of a distribution is the portion of the distribution corresponding to the large or extreme values of the random variable under study. Because extreme events are rare, a probabilistic framework is used, requiring a quantitative estimation of the asymptotic behavior of the losses. The extreme events can be treated by the asymptotic theory discovered by Fisher and Tippett (1928), Gnedenko (1943) and Gumbel (1958) using three extreme-value types of distribution. The work of Embrechts et al. (1997), Coles (2001), Kotz and Nadarajah (2000), Beirlant et al. (2004), McNeil et al. (2005) and Reiss and Thomas (2008) has been extensively cited as examples of progress in statistical modeling of extreme values. The Block Maxima (BM) method and Peak over Thresholds (PoT) method are the two primary methods for univariate EVT.

In the BM method, Generalized Extreme Value (GEV) distribution is used as a limiting distribution for the maxima in a given period of time called a "block." It can be written as:

$G(z)=\exp \left\{-\left[1+\gamma\left(\frac{z-\mu}{\sigma}\right)\right]_{+}^{-1 / \gamma}\right\}$ if $1+\gamma\left(\frac{z-\mu}{\sigma}\right)>0$

This formula incorporates Gumbel (shape parameter $\gamma \rightarrow 0$ ), Frechet $(\gamma>0)$ and Weibull $(\gamma<0)$ distributions within. Provided that the proper scaling is performed, the distribution of the block maximum losses can be approximated by one of these three distributions. GEV distribution can be used for per occurrence PML calculation.

The PoT method utilizes GPD to model the exceedances over a sufficiently large threshold u. GPD is an asymptotically motivated distribution and serves as an approximation of $\mathrm{F}_{\mathrm{u}}=\operatorname{Pr}(\mathrm{X}<\mathrm{X} / \mathrm{X}>\mathrm{u})$ for a broad class of models $\mathrm{F}$. Let $\mathrm{y}$ denote the exceedance over $\mathrm{u}$, that is, $\mathrm{y}=\mathrm{x}-\mathrm{u}$. The $\mathrm{CDF}$ of the GPD is defined as: 
$W(y)=1-\left[1+\xi\left(\frac{y}{\sigma}\right)\right]^{-1 / \xi}$ for $\xi \neq 0, W(y)=1-e^{-\frac{y}{\sigma}}$ for $\xi=0$.

GPD posses modeling flexibility and depending on the parameter values, it can be heavytailed or light-tailed. At $\xi=0$, GPD becomes an exponential distribution. If $\xi<0$, $\mathrm{x}$ is

capped at $\mathrm{u}-\frac{\sigma}{\xi}$ while for $\xi>0$, $\mathrm{x}$ is potentially unbounded. GPD with $\xi \geq \frac{1}{2}$ has infinite variance and is unusual in statistical applications.

In a three-parameter form, this can be rewritten as:

$W(x)=1-\left[1+\xi\left(\frac{x-u}{\sigma}\right)\right]^{-1 / \xi}$ for $\xi \neq 0, W(x)=1-e^{-\frac{x-u}{\sigma}}$ for $\xi=0$

The theoretical foundations of PoT method and properties of GPD can be found in Appendix C.

\subsection{Risk Measures}

The purpose of cat models is to assess cat risks to facilitate operational and strategic decisions. The losses generated by a cat model can be summarized and extracted into risk measures to quantify risk exposure. The inferences from a fitted heavy-tailed model are usually made by tail-based risk measures such as extreme quantiles and mean tail loss. They are usually accompanied by an associated return period, which is defined as $T=\frac{1}{1-p}$, where $(1-\mathrm{p})$ is the exceedance probability. These risk measures serve as a basis for risk assessment and are typical evaluation methods for (re)insurance premiums and regulatory capital requirements. For example, both the pricing and the design of layer structures of an insurer's reinsurance programs require PMLs at various attainment and exhaustion probabilities. Moreover, investors, regulators and rating agencies are 
interested in the company's ability to buffer against adverse events which is expressed as the economic capital at a particular (high) probability level.

My study provided asymptotic tail probabilities that could be used to estimate certain risk measures such as PML, another name for VaR, and TVaR. The $\mathrm{PML}_{\mathrm{p}}$ of the random variable $\mathrm{X}$ is $\mathrm{p}$ quantile, the 100pth percentile, of the distribution of $\mathrm{X}$, that is, $\mathrm{p}\left(\mathrm{X}<\mathrm{PML}_{\mathrm{p}}\right)=\mathrm{p}$. In practice, if an insurer holds capital equivalent to $\mathrm{PML}_{\mathrm{p}}$, it is supposed to absorb $100 \mathrm{p} \%$ of the potential outcomes and thus stays possibly solvent under a certain adverse event. In this context, when $\mathrm{p}=99.5 \%$, it means there is a very small chance $(0.5 \%)$ that Florida will encounter hurricane loss of $\mathrm{PML}_{0.995}$ or a one in 200 -year event. In the parametric method, PML can be calculated as a function of parameters by the inverse method. The return period is the waiting time for the exceedance of the particular quantile. The $\mathrm{p}$ quantile of $\mathrm{F}$ is defined by quantile function:

$F^{-1}(p):=\inf \{x: F(x) \geq p\}$, where $0<\mathrm{p}<1$.

Take the lognormal distribution for an example: the CDF is $F(x)=\Phi\left(\frac{\ln x-\mu}{\sigma}\right)$; taking the inverse, we get $P M L_{p}=F^{-1}(p)=e^{\mu+\propto \Phi^{-1}(p)}$.

For GPD, a distribution function of the whole data range needs to be developed. $F(x)=\left(1-F_{n}(u)\right) W_{\xi, u, \sigma}(x)+F_{n}(u)$ is used to approximate $\mathrm{F}(\mathrm{x})$ in which $F_{n}(u)$ is the empirical cumulative probability of the threshold $u$ using the entire data and is estimated as $\left(\mathrm{N}-\mathrm{N}_{\mathrm{u}}\right) / \mathrm{N}$, where $\mathrm{N}$ is the sample size and $\mathrm{N}_{\mathrm{u}}$ denotes the number of observations above the chosen threshold $u$. The PML for a given exceedance probability $q(q=1-p)$ of GPD is given by: 
$P M L_{p}=V a R_{p}=u+\frac{\sigma}{\xi}\left[\left(\frac{N}{N_{u}} q\right)^{-\xi}-1\right]$

The use of PML (VaR) is pervasive in the industry because it is easy to interpret and communicate among different users. However, PML has two main disadvantages: 1) it violates the subadditivity property of coherent risk measure ${ }^{3} 2$ ) measuring only a single quantile, it does not observe capital loss above it and thus could lead to underestimation of the potential losses (Klugman et al., 2008). As a coherent measure, Tail-Value-at-Risk (TVaR, also referred to as CVaR and expected shortfall) is gaining popularity as it adds the expected loss above VaR to the quantile:

$$
T \operatorname{VaR}_{p}=E\left(X \mid X>V a R_{p}\right)=V a R_{p}+E\left(X-\operatorname{VaR}_{p} \mid X>V a R_{p}\right) \quad \text { with associated return }
$$
period $1 / \mathrm{q}$.

The second term is known as the mean excess function of GPD and for $\xi<1$

$T V a R_{p}=\operatorname{VaR}_{p}+\frac{\sigma+\xi\left(V a R_{p}-u\right)}{1-\xi}=\frac{V a R_{p}}{1-\xi}+\frac{\sigma-\xi u}{1-\xi}$ (Brodin and Rootzen, 2009).

Inferences based on cat models are very sensitive to the largest observed losses and the introduction of a new extreme loss to the dataset may have a substantial impact. Hence, it is problematic to rely on any one single statistic to summarize the risk contained in the data. In practice, stress testing and worst case scenario are used to supplement PML.

\footnotetext{
${ }^{3}$ A coherent risk measure is a risk measure $\rho(\mathrm{X})$ that has the following four properties for any two loss random variables $\mathrm{X}$ and $\mathrm{Y}$ :

1. Subadditivity: $\rho(X+Y) \leq \rho(X)+\rho(Y)$.

2. Monotonicity: If $X \leq Y$ for all possible outcomes, then $\rho(X) \leq \rho(Y)$.

3. Positive homogeneity: For any positive constant $c, \rho(c X)=c \rho(X)$.

4. Translation invariance: For any positive constant $c, \rho(X+c)=\rho(X)+c$.
} 


\subsection{Non-parametric Method}

Unlike parametric models discussed in 2.1 and 2.2, non-parametric method assumes the empirical loss distribution to be a close substitute for the population loss distribution, free from any parametric constrains. PML can be produced non-parametrically through order statistics. To estimate PML of the $100 \mathrm{p}^{\text {th }}$ percentile, the $\mathrm{k}^{\text {th }}$ order statistic, $\mathrm{X}_{\mathrm{k}}$, is used. $\mathrm{K}$ is determined by sample size $\mathrm{N}$ multiplied by $\mathrm{p}$. If the result is not an integer, the smoothed empirical estimate is applied to interpolate two adjacent order statistics through $\mathrm{PML}_{\mathrm{p}}=(1-\mathrm{h}) \mathrm{x}_{\mathrm{j}}+\mathrm{hx}_{\mathrm{j}+1}$ where $\mathrm{j}=[(\mathrm{N}+1) \mathrm{p}]$ and $\mathrm{h}=(\mathrm{N}+1) \mathrm{p}-\mathrm{j}$; here [.] indicates the greatest integer function (Klugman et al., 2008). This method, however, is not applicable for $\mathrm{PML}_{\mathrm{p}}$ where $\mathrm{p}>\mathrm{N} /(\mathrm{N}+1)$.

The approximate $95 \%$ confidence interval for $\mathrm{PML}_{\mathrm{p}}$ is given by $\left(\mathrm{X}_{\mathrm{r}}, \mathrm{X}_{\mathrm{s}}\right)$. The large sample assumes normal approximation to obtain $\mathrm{r}$ and $\mathrm{s}$ as

$$
\begin{aligned}
& r=N p-1.96 \sqrt{N p(1-p)} \\
& s=N p+1.96 \sqrt{N p(1-p)} .
\end{aligned}
$$

In case any value of $r$ and $s$ is not an integer, the smoothed empirical estimate is used.

The exact confidence interval ${ }^{4}$ can be calculated which does not rely on the normality assumption. The values of $\mathrm{r}$ and $\mathrm{s}$ can be found through numerical analysis with $\mathrm{r}<\mathrm{s}$ such that

\footnotetext{
${ }^{4}$ Disclosure in Form A11. Reference from Approximate distributions of order statistics with applications to nonparametric statistics by R.D. Reiss
} 


$$
\begin{aligned}
& p\left(X_{(r)}<P M L_{p}<X_{(s)}\right) \\
& =p\left(P M L_{p}<X_{(s)}\right)-p\left(P M L_{p}<X_{(r)}\right) \\
& =\sum_{i=1}^{s-1}\left(\begin{array}{l}
N \\
i
\end{array}\right) p^{i}(1-p)^{N-i}-\sum_{i=1}^{r-1}\left(\begin{array}{l}
N \\
i
\end{array}\right) p^{i}(1-p)^{N-i} \\
& =\sum_{i=r}^{s-1}\left(\begin{array}{l}
N \\
i
\end{array}\right) p^{i}(1-p)^{N-i} \approx 0.95
\end{aligned}
$$

If the solution from the computer search is not unique, the pair of $r$ and $s$ that minimizes s-r is selected to give the narrowest interval. 


\section{Chapter 3}

\section{PARAMETERS ESTIMATION}

In parametric distribution fitting, the data are assumed to follow some specific parametric models. The parameters such as $\mu, \sigma$ and $\alpha$ condense the important information of the data that determine the probability distributions. A wide variety of methods can be used to estimate the parameters of a distribution. Maximum Likelihood Estimation (MLE) is commonly used mainly because of the desirable asymptotic properties of consistency, efficiency and normality. Other methods include probability weighted moment estimation, $\mathrm{L}$ estimation and Bayesian estimation. The advantages and disadvantages of major estimation methods for GPD are discussed in details in Bermudez and Kotz (2010).

For heavy-tailed distributions, the shape parameter $\alpha$ provides an indication of the tail heaviness. In EVT, $\alpha$ (the same as $\xi$ in 2.1.2) is also known as Pareto index or extreme value index. Estimating $\alpha^{*}$ of a Pareto law regarding to the regular variation is in essence equivalent to calculating the semiparametric estimator derived under the sole condition of

$F \in \operatorname{MDA}\left(H_{\xi}\right)$, because $\alpha^{*}=\frac{1}{\xi}\left(\alpha^{*}\right.$ is shape parameter, so is $\left.\xi\right)$. Hill, Pickands and QQ estimation methods can be used to estimate the asymptotic behavior of a sample on the basis of the tail characteristics. The details of these techniques can be found in Resnick (2007). They are called semi-parameteric estimators because there is no need to assume a probabilistic distribution (Bernardara et al., 2008). Furthermore, covariates in the real data can be incorporated into $\alpha$ through tail index regression model, enabling the shape parameter to better capture the characteristics of the sample (Wang and Tsai, 2009). 
Estimators like MLE, Method of Moments (MoM) and probability weighted moment lack robustness, making the inference vulnerable to a single extreme (Juarez and Schucany, 2004). Furthermore, MLE may encounter a convergence problem in optimizing the log likelihood function and moment equations may not have closed form in MoM. In recent years, Dupuis' (1998) optimally-based robust estimator, Peng and Welsh's (2002) medians estimator and Juarez and Schucany's (2004) minimum density power divergence estimator were proposed. Moreover, robust estimators based on t-score moments, "generalized median", "trimmed mean" type are developed to avoid subjective assumption of a theoretical distribution (Stehlik et al., 2010).

For simplicity and the purpose of preliminary data analysis, MoM was used in this study. Due to the lack of the literature on estimating parameters of $\mathrm{TB}$, this distribution was removed from my analysis. Because the focus of the study was to explore the potential of PoT method, GPD was extensively analyzed using MoM and MLE. All the computations were done by R. 


\section{Chapter 4}

\section{APPLICATION}

\subsection{Losses Generated from a Stochastic Hurricane Set}

In order to compute per occurrence PML, annual maximum losses were used. Losses of the same year were added together for per aggregate PML analysis. Some descriptive statistics are given in Table 4.1.
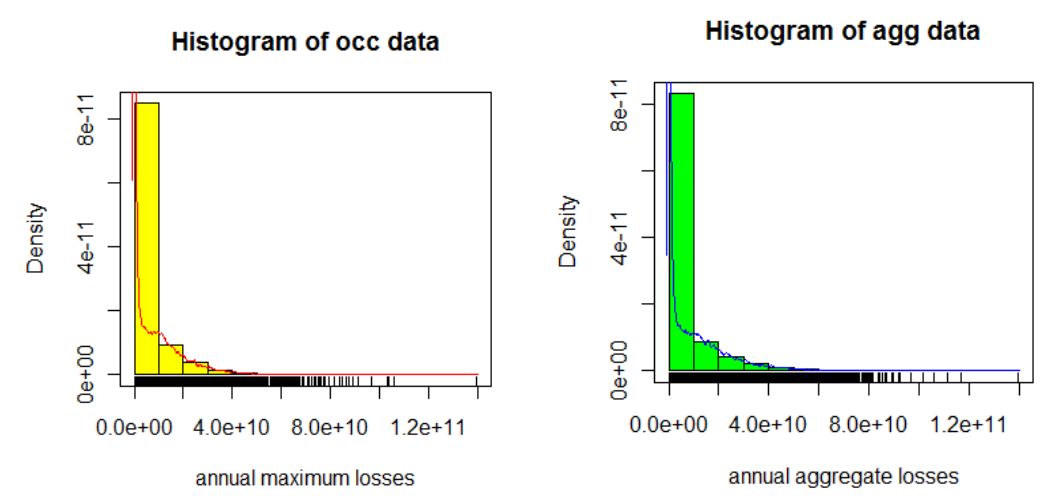

Figure 4.1 Histograms for annual maximum losses (left) and annual aggregate loss (right)

Table 4.1 Selected descriptive statistics

\begin{tabular}{|c|c|c|c|c|}
\hline data & mean & std & skewness & kurtosis \\
\hline Occ & $3,952,696,011$ & $8,562,466,767$ & 3.193 & 17.043 \\
\hline Agg & $4,596,495,840$ & $9,996,759,996$ & 3.076 & 15.047 \\
\hline
\end{tabular}

Outliers can be seen in the far right tail in Figure 4.1. Both sets of the data have high variances accompanying the large means with the corresponding coefficient of variance of 2.166 and 2.175 respectively. Positive skewness implies that the upper tail is more pronounced than the lower tail. Both kurtosis are much larger than 3, an indication of a longer and fatter tail than the normal distribution.

The Skewness-Kurtosis plot in Figure 4.2 shows the range of skewness and kurtosis values a distribution can take. It provides a helpful tool for the exploratory data analysis. 
The simulated data set lies in the beta regime. Beta distribution is known for its flexibility in shapes, consequently covering a wide variety of skewness-kurtosis sets in the plot. In this sense, it is reasonable to presume Beta as the parent distribution of the underlying data. If a distribution fits the data, the parent distribution will at least fit it (Dutta and Perry, 2006). The bootstrap samples give a possible range of skewness-kurtosis values, some of which are close to Gamma distribution. Overall, it is observed from the location of the data that for the same skewness, Gamma has higher kurtosis, heavier tail, than the empirical distribution. Additionally, the annual maximum losses have much heavier tail than Exponential and lighter tail than Lognormal distribution.

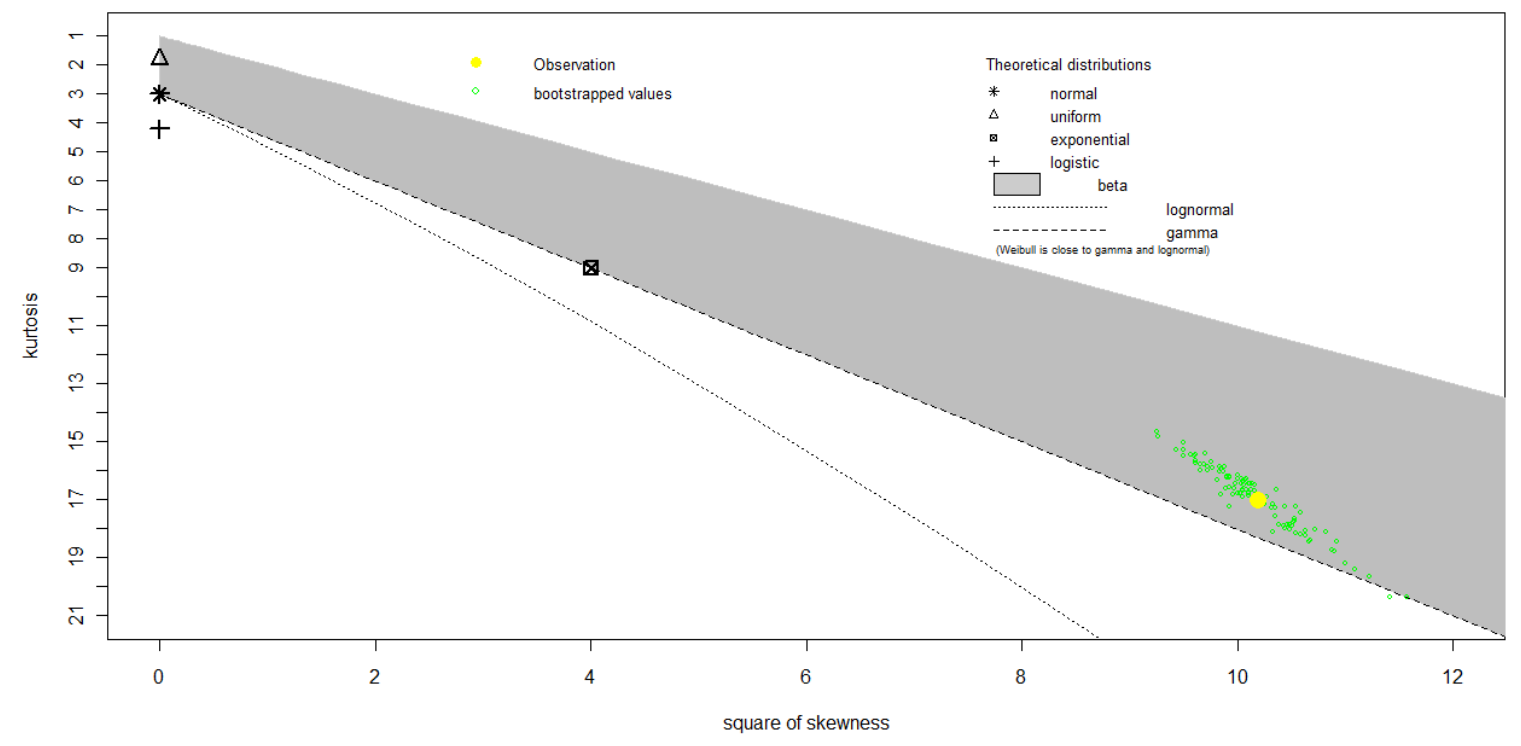

Figure 4.2 Skewness-Kurtosis plot for per occ data with 100 bootstrap samples

Similar conclusions can be drawn for per agg data. The sample skewness-kurtosis and its 100 bootstrap values lie completely within the Beta region, which is another justification for TB to be considered for modeling FPHLM data. GPD is an appealing choice for 
fitting the hurricane losses because it belongs to TB Family and is frequently used as a model for extremes.

However the threshold selection of GPD possesses great difficulty as a result of bias-andvariance tradeoff. Ideally, the threshold is selected to extract maximum amount of information from the data while remaining in the asymptotic zone (Bernardara et al., 2008). So far, there has been no well-defined theory in optimizing the choice of threshold. Traditional methods include examining various diagnostic plots such as mean excess plots, parameter stability plots, QQ plots and quantile plots. A simple diagnostic tool is to look for linearity in the sample mean excess plot since if the data follows GPD and provided $\xi<1, \mathrm{E}(\mathrm{X}-\mathrm{u} / \mathrm{X}>\mathrm{u})$ is a linear function of $\mathrm{u}$.

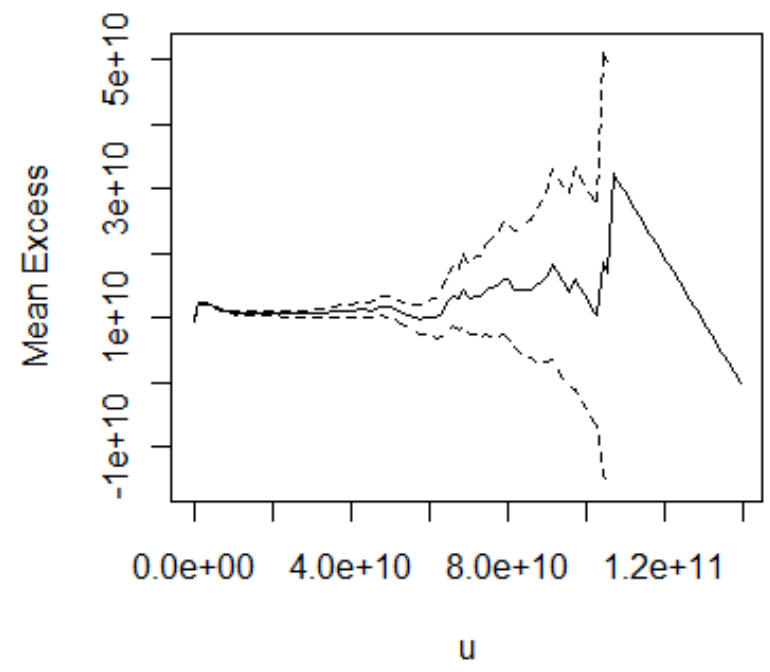

Figure 4.3 Mean Excess Plot for per occ data with 95\% confidence limits 
The variability of the mean excess plot of per occurrence data in Figure 4.3 increases with the increasing thresholds. Upon closer examination, thresholds from 10 billion to 50 billion were identified as a stable range with less uncertainty.
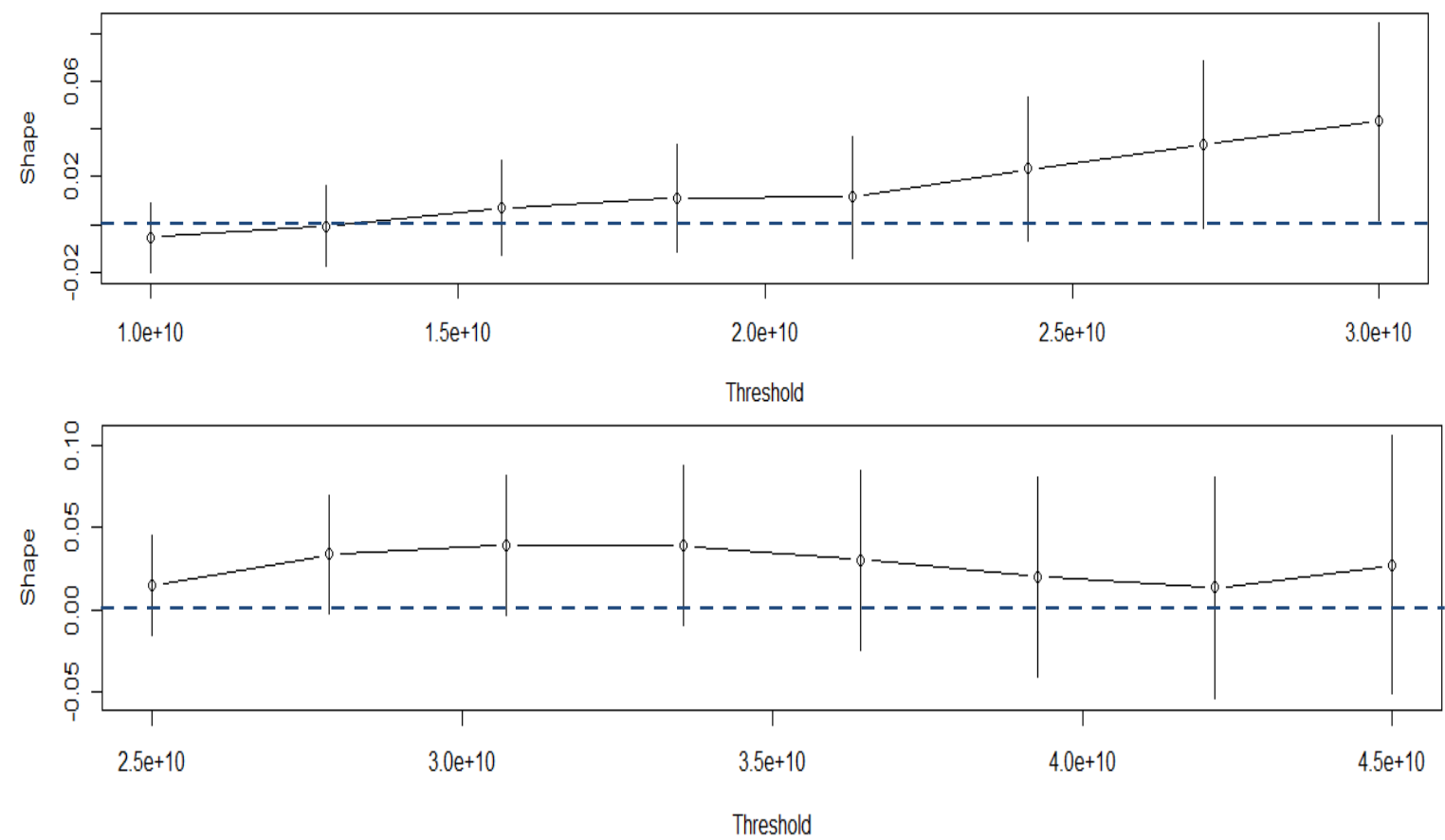

Figure 4.4 Shape parameter variability plot for per occ data, $\xi=0$ (dashed line)

Figure 4.4 shows that the shape parameters between 10 billion and 30 billion are relatively stable with an increasing trend in the point estimates ranging from 0 to 0.04 . From 25 billion to 45 billion, the estimate varies between 0 and 0.05 . Most of the selected confidence intervals include zero. Several thresholds were chosen as shown in Table 4.2 and the MLE shape parameters all yield values near zero, which indicates an exponential tail. Thresholds of 40 billon, 35 billion, 30 billion and 20 billion have the smaller standard errors with respect to their point estimates. The threshold of 20 billion was chosen for including large amount of data in the analysis. 
Table 4.2 Shape parameter comparison, per occ data

\begin{tabular}{|c|c|c|c|c|c|}
\hline $\mathrm{u}$ & shape & std error & ratio & \# exceed & \% exceed \\
\hline $10,000,000,000$ & -0.005 & 0.008 & 1.38 & 8272 & $16.16 \%$ \\
\hline $15,000,000,000$ & 0.006 & 0.010 & 1.57 & 5267 & $10.29 \%$ \\
\hline $20,000,000,000$ & 0.012 & 0.012 & 0.99 & 3309 & $6.46 \%$ \\
\hline $25,000,000,000$ & 0.015 & 0.016 & 1.05 & 2056 & $4.02 \%$ \\
\hline $30,000,000,000$ & 0.043 & 0.021 & 0.49 & 1300 & $2.54 \%$ \\
\hline $35,000,000,000$ & 0.292 & 0.037 & 0.13 & 792 & $1.55 \%$ \\
\hline $40,000,000,000$ & 0.302 & 0.047 & 0.16 & 500 & $0.98 \%$ \\
\hline $45,000,000,000$ & 0.027 & 0.040 & 1.46 & 316 & $0.62 \%$ \\
\hline $50,000,000,000$ & -0.015 & 0.043 & 2.85 & 194 & $0.38 \%$ \\
\hline
\end{tabular}

Figure 4.5 is another way to demonstrate that the confidence intervals of $\xi$ at different thresholds contain zero. The interval width increases to reflect the uncertainty as the threshold increases and sample size decreases. For all the thresholds in the range, the hypothesis of an underlying exponential distribution was not rejected. Particularly at $\mathrm{u}=20,000,000,000$, the likelihood ratio statistic was 0.5614808 which is smaller than 3.841459, the chi-square critical value. There is sufficient evidence that the exceedances resemble an exponential distribution.

Threshold

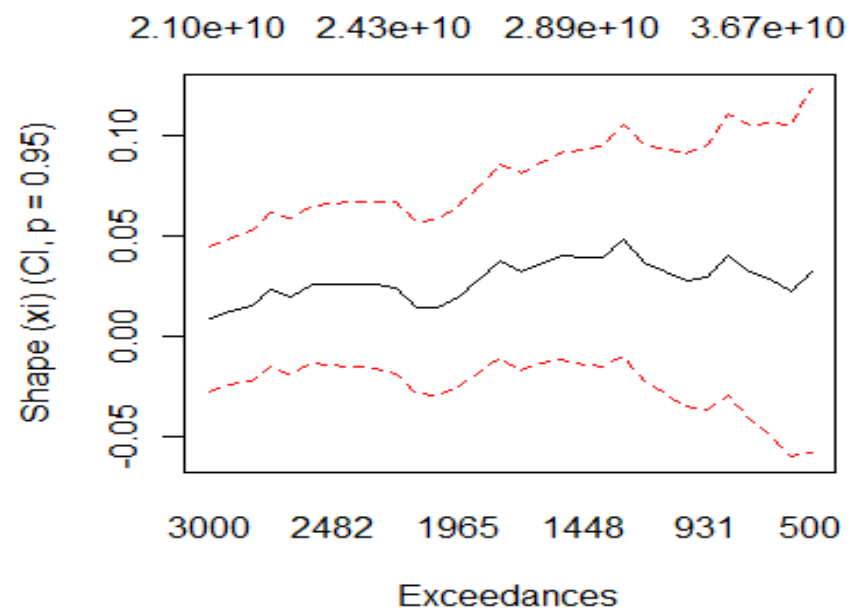

Figure 4.5 Shape estimates with 95\% confidence limits for a selected threshold range, per occ 
The standard method for constructing confidence interval is based on asymptotic normality of MLE of the parameter. The $95 \%$ confidence interval for the shape parameter when $\mathrm{u}=20,000,000,000$ is $(-0.0119,0.03622)$. The profile likelihood confidence interval derived from an asymptotic $\chi^{2}$ distribution of the likelihood-ratio test statistic is considered to be more robust in small samples. The $95 \%$ profile likelihood confidence interval is approximately $(-0.01073,0.03749)$. Both intervals contain zero.

The same techniques were applied for the threshold selection of annual aggregate losses. The shape parameters are relatively stable at values slightly below zero.

Table 4.3 Shape parameter comparison, per agg

\begin{tabular}{|c|c|c|c|c|c|}
\hline $\mathrm{u}$ & Shape & ratio & ratio & \# Exceed & $\%$ \\
\hline $10,000,000,000$ & -0.052 & 0.007 & 0.127 & 9144 & $16.9 \%$ \\
\hline $15,000,000,000$ & -0.033 & 0.008 & 0.251 & 6463 & $12.0 \%$ \\
\hline $20,000,000,000$ & -0.037 & 0.010 & 0.267 & 4380 & $8.1 \%$ \\
\hline $25,000,000,000$ & -0.036 & 0.012 & 0.333 & 2971 & $5.5 \%$ \\
\hline $30,000,000,000$ & -0.027 & 0.015 & 0.549 & 2022 & $3.7 \%$ \\
\hline $35,000,000,000$ & -0.036 & 0.017 & 0.482 & 1348 & $2.5 \%$ \\
\hline $40,000,000,000$ & -0.015 & 0.022 & 1.487 & 914 & $1.7 \%$ \\
\hline $45,000,000,000$ & 0.201 & 0.038 & 0.191 & 598 & $1.1 \%$ \\
\hline $50,000,000,000$ & -0.021 & 0.032 & 1.527 & 390 & $0.7 \%$ \\
\hline
\end{tabular}

Threshold

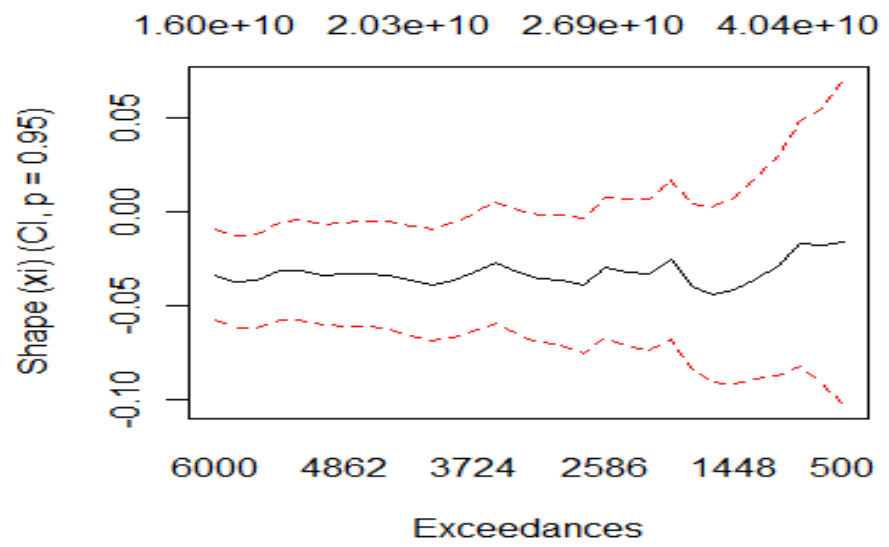

Figure 4.6 Shape estimates with 95\% confidence limits for a selected threshold range, per agg 
The shape parameter $\xi$ determines the convexity of the relationship between the extreme quantile $\mathrm{x}$ and the return period $\mathrm{T}(\mathrm{x})$. Larger $\xi$ corresponds to a more convex curve in the return level plot. The fact that the selected thresholds produced similar $\xi$ indicates that there should be no material difference in the generated VaR and TVaR estimates, which is validated in Table 4.4 .

Table 4.4 Risk measures for different thresholds, per agg (in 000s)

\begin{tabular}{|c|c|c|c|c|c|c|}
\hline & \multicolumn{3}{|c|}{ VaR } & \multicolumn{3}{c|}{ TVaR } \\
\hline p $\backslash \mathrm{u}$ & $10,000,000$ & $15,000,000$ & $20,000,000$ & $10,000,000$ & $15,000,000$ & $20,000,000$ \\
\hline 0.9 & $17,217,620$ & $17,341,146$ & $17,260,386$ & $29,988,506$ & $29,916,053$ & $29,906,938$ \\
\hline 0.95 & $26,391,866$ & $26,242,848$ & $26,246,177$ & $38,668,021$ & $38,533,383$ & $38,555,438$ \\
\hline 0.97 & $32,924,716$ & $32,673,994$ & $32,714,643$ & $44,848,578$ & $44,759,081$ & $44,781,103$ \\
\hline 0.975 & $35,210,711$ & $34,943,244$ & $34,992,311$ & $47,011,298$ & $46,955,838$ & $46,973,276$ \\
\hline 0.99 & $46,346,474$ & $46,143,319$ & $46,197,106$ & $57,546,552$ & $57,798,118$ & $57,757,487$ \\
\hline 0.995 & $54,392,295$ & $54,393,682$ & $54,411,136$ & $65,158,493$ & $65,784,916$ & $65,663,195$ \\
\hline 0.997 & $60,121,610$ & $60,354,260$ & $60,324,047$ & $70,578,847$ & $71,555,079$ & $71,354,158$ \\
\hline 0.999 & $71,892,503$ & $72,838,032$ & $72,648,545$ & $81,714,981$ & $83,640,047$ & $83,216,043$ \\
\hline
\end{tabular}

Value of 20 billion was selected as the threshold for annual aggregate losses. MLE and MoM produced close parameter estimates, therefore similar risk measures.

Table 4.5 MLE and MoM comparison of extreme risk measures (in 000s)

\begin{tabular}{|c|c|c|c|c|c|c|}
\hline & \multicolumn{2}{|c|}{ VaR } & \multicolumn{2}{c|}{ TVaR } & \multicolumn{2}{c|}{ difference } \\
\hline $\mathrm{p}$ & $\mathrm{MLE}$ & $\mathrm{MoM}$ & $\mathrm{MLE}$ & $\mathrm{MoM}$ & $\mathrm{V}$ & $\mathrm{TVR}$ \\
\hline 0.9 & $17,264,210$ & $17,260,465$ & $29,914,821$ & $29,909,651$ & $0.02 \%$ & $0.02 \%$ \\
\hline 0.95 & $26,241,775$ & $26,246,593$ & $38,572,069$ & $38,560,775$ & $-0.02 \%$ & $0.03 \%$ \\
\hline 0.97 & $32,712,115$ & $32,716,379$ & $44,811,548$ & $44,789,368$ & $-0.01 \%$ & $0.05 \%$ \\
\hline 0.975 & $34,992,026$ & $34,994,730$ & $47,010,112$ & $46,982,782$ & $-0.01 \%$ & $0.06 \%$ \\
\hline 0.99 & $46,220,125$ & $46,204,582$ & $57,837,594$ & $57,774,730$ & $0.03 \%$ & $0.11 \%$ \\
\hline 0.995 & $54,464,517$ & $54,424,149$ & $65,787,827$ & $65,687,871$ & $0.07 \%$ & $0.15 \%$ \\
\hline 0.997 & $60,406,441$ & $60,342,031$ & $71,517,744$ & $71,385,135$ & $0.11 \%$ & $0.19 \%$ \\
\hline 0.999 & $72,811,287$ & $72,679,624$ & $83,479,987$ & $83,262,782$ & $0.18 \%$ & $0.26 \%$ \\
\hline
\end{tabular}


Parametric methods have an advantage of producing distributions of risk measures to gain computational efficiency. Exponential (noted as exp in the tables below), Gamma, Lognormal (lnorm), Pareto, Loglogistic along with Generalized Pareto (GP) were selected to fit the data through MoM. Per occurrence PML and TVaR were calculated and the results were compared empirically. Table 4.6 and 4.7 show selected points that may be of interest to practitioners.

Table 4.6 Per Occurrence PML (in 000s)

\begin{tabular}{|c|c|c|c|c|c|c|c|c|}
\hline $\mathrm{EP}$ & $\mathrm{T}$ & $\exp$ & gamma & lnorm & Pareto & loglogistic & GP & empirical \\
\hline 0.1 & 10 & $9,101,419$ & $11,950,279$ & $8,979,035$ & $8,983,645$ & $6,578,655$ & $14,859,252$ & $14,732,953$ \\
\hline 0.05 & 20 & $11,841,219$ & $20,025,368$ & $14,497,922$ & $13,710,802$ & $8,237,445$ & $22,145,056$ & $22,246,330$ \\
\hline 0.03 & 33 & $13,860,357$ & $26,620,631$ & $19,789,601$ & $18,119,353$ & $9,666,663$ & $27,558,670$ & $27,687,021$ \\
\hline 0.025 & 40 & $14,581,019$ & $29,075,849$ & $21,967,506$ & $19,920,063$ & $10,227,667$ & $29,500,017$ & $29,601,406$ \\
\hline 0.01 & 100 & $18,202,838$ & $42,032,928$ & $35,614,096$ & $31,211,687$ & $13,537,061$ & $39,329,997$ & $39,020,780$ \\
\hline 0.005 & 200 & $20,942,638$ & $52,359,682$ & $49,489,024$ & $42,907,992$ & $16,702,136$ & $46,848,076$ & $46,492,754$ \\
\hline 0.003 & 333 & $22,961,776$ & $60,186,001$ & $62,085,760$ & $53,815,977$ & $19,489,271$ & $52,434,278$ & $53,078,209$ \\
\hline 0.001 & 1000 & $27,304,257$ & $77,490,560$ & $97,527,236$ & $86,210,060$ & $27,141,761$ & $64,580,880$ & $63,592,927$ \\
\hline
\end{tabular}

Table 4.7 Per Occurrence TVaR (in 000s)

\begin{tabular}{|c|c|c|c|c|c|c|c|c|}
\hline $\mathrm{EP}$ & $\mathrm{T}$ & $\exp$ & gamma & lnorm & Pareto & loglogistic & GP & empirical \\
\hline 0.1 & 10 & $13,054,115$ & $24,610,074$ & $20,350,288$ & $18,763,717$ & $9,515,855$ & $25,465,429$ & $25,440,556$ \\
\hline 0.05 & 20 & $15,793,915$ & $33,793,559$ & $29,422,420$ & $26,557,215$ & $11,742,111$ & $32,851,906$ & $32,849,693$ \\
\hline 0.03 & 33 & $17,813,053$ & $40,985,343$ & $37,820,843$ & $33,825,440$ & $13,653,521$ & $38,340,323$ & $38,280,361$ \\
\hline 0.025 & 40 & $18,533,715$ & $43,621,017$ & $41,218,604$ & $36,794,206$ & $14,396,976$ & $40,308,496$ & $40,215,680$ \\
\hline 0.01 & 100 & $22,155,534$ & $57,300,023$ & $61,989,853$ & $55,410,311$ & $18,650,363$ & $50,274,304$ & $50,183,058$ \\
\hline 0.005 & 200 & $24,895,334$ & $68,026,664$ & $82,505,907$ & $74,693,599$ & $22,416,001$ & $57,896,265$ & $57,984,982$ \\
\hline 0.003 & 333 & $26,914,472$ & $76,092,669$ & $100,794,948$ & $92,677,212$ & $25,398,399$ & $63,559,655$ & $63,888,704$ \\
\hline 0.001 & 1000 & $31,256,953$ & $93,802,074$ & $151,159,698$ & $146,084,202$ & $31,346,447$ & $75,874,095$ & $75,252,317$ \\
\hline
\end{tabular}

Generally speaking, Gamma and Lognormal are not considered as heavy-tailed distributions, yet at the very extreme quantile points, they appear to overestimate the empirical quantiles. Both Exponential and Loglogistic underestimate the empirical. This 
result agrees with the conclusions from Skewness-Kurtosis plot. Among the selected distributions, Generalized Pareto $(u=20,000,000,000)$ fits the data the best and Gamma ranks the next. Note as the exceedance probability (EP) approaches one, the empirical VaR and TVaR converge to the data's maximum 139,359,000,000. The TVaR value from Pareto distribution exceeds the maximum at 891.47 year and that of Lognormal attains it sometime between the 333 and the 1000 return period.

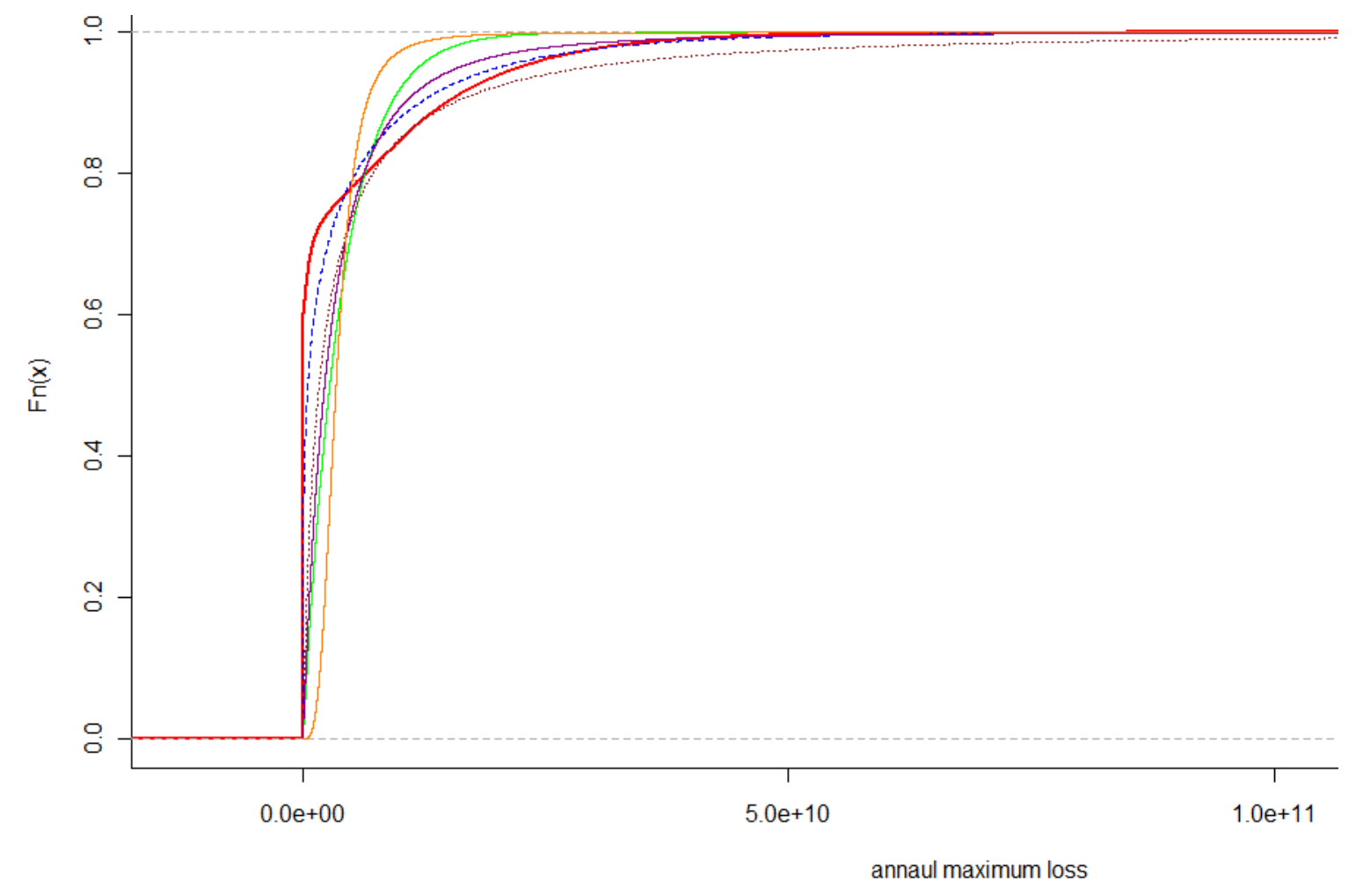

Figure 4.7 CDF comparison for modeling annual maximum losses

\begin{tabular}{|ll|}
\hline Empirical & \\
\cline { 2 - 2 } Gamma & \\
Lnorm & \\
Pareto & \\
Loglogistic & \\
\hline
\end{tabular}

The five distributions fitted to the whole data are compared with the empirical distribution at the full domain in Figure 4.7. Typically, the selected distributions underestimate the empirical at the left tail and roughly between 10 billion and 25 billion, 
all of them overestimate the empirical losses. Exponential and Loglogistic approach one much faster than the others, which agrees with the results observed in Table 4.6 and 4.7. In estimating PML for the loss distribution, one of the common techniques actuaries use is the mixed model where a weighted average of the risk measures produced by different models is used to reflect the characteristics of empirical data.

QQ plot in Figure 4.8 suggests that the GPD $(u=20,000,000,000$ and $\xi=0.0136)$ fits the empirical data well most of the time and overestimates several points in farther tail.

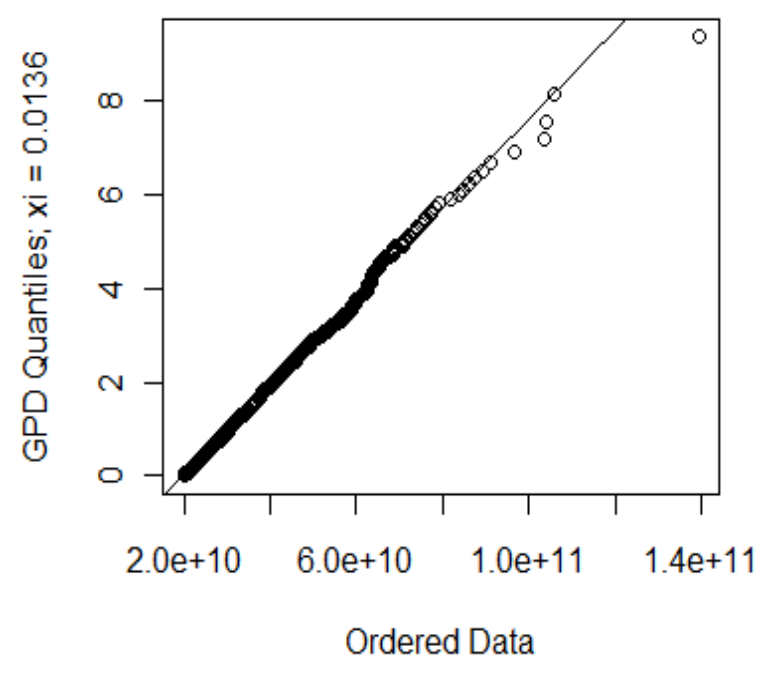

Figure 4.8 QQ plot for per occurrence loss using GPD 
Threshold

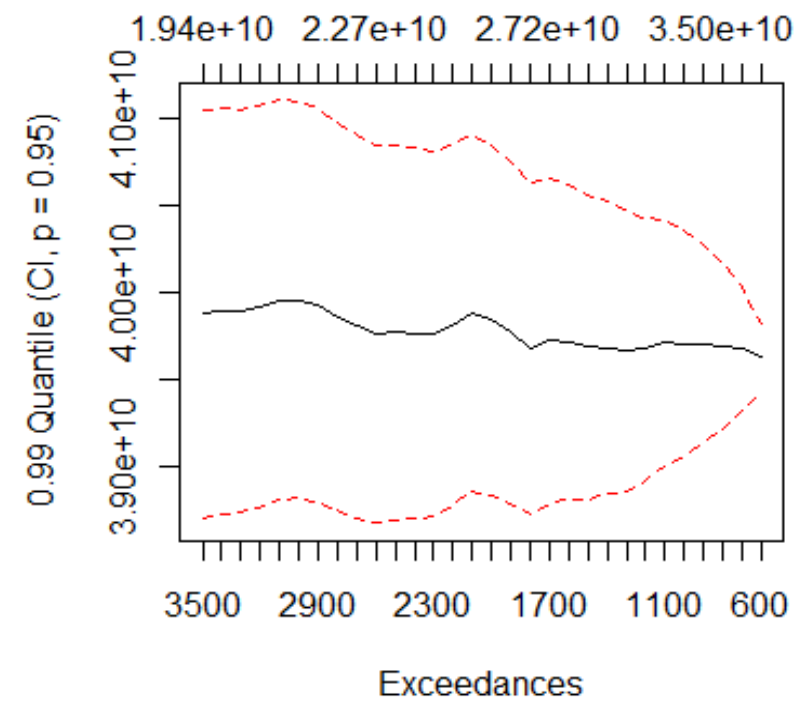

Figure $4.995 \%$ confidence intervals for $99^{\text {th }}$ percentile with varying threshold, per occ High quantile such as a 100-year return level as shown in Figure 4.9 is insensitive to the threshold choices, which indicates low threshold risk in this dataset. However, the large interval width suggests greater uncertainty in the estimation. The decrease in the confidence width can be partly explained by the better satisfied condition for EVT application.

For the aggregate data set, a threshold of 20 billion was chosen after considering sample size and shape parameter stability. The MLE for shape parameter was -0.037 with standard error of 0.0524 compared with a point estimate of -0.039 using MoM. The $95 \%$ profile likelihood confidence interval is $(-0.05564,-0.01688)$. 


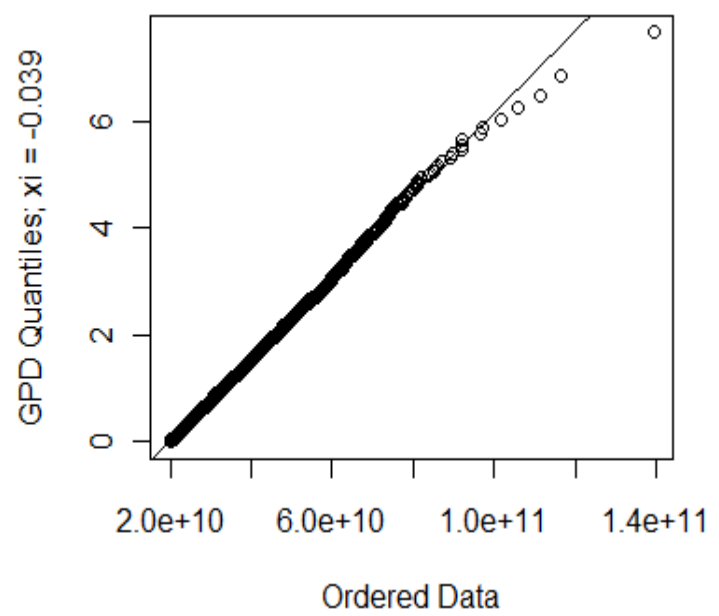

Figure 4.10 QQ plot for per aggregate loss using GPD

The QQ plot indicates that GPD tends to overestimate higher quantiles produced by the simulated annual loss data. A comparison of results for PML and TVaR is made in Table 4.8 and 4.9 respectively.

Table 4.8 Per Aggregate PML (in 000s)

\begin{tabular}{|c|c|c|c|c|c|c|c|c|}
\hline $\mathrm{EP}$ & $\mathrm{T}$ & $\exp$ & gamma & lnorm & Pareto & loglogistic & GP & empirical \\
\hline 0.1 & 10 & $10,583,823$ & $13,898,523$ & $10,440,507$ & $10,443,985$ & $7,910,185$ & $17,260,386$ & $17,358,318$ \\
\hline 0.05 & 20 & $13,769,871$ & $23,337,359$ & $16,872,901$ & $15,945,795$ & $10,150,511$ & $26,246,177$ & $26,270,450$ \\
\hline 0.03 & 33 & $16,117,879$ & $31,052,794$ & $23,044,945$ & $21,079,365$ & $12,121,228$ & $32,714,643$ & $32,560,173$ \\
\hline 0.025 & 40 & $16,955,919$ & $33,925,918$ & $25,586,143$ & $23,176,814$ & $12,903,797$ & $34,992,311$ & $34,996,373$ \\
\hline 0.01 & 100 & $21,167,646$ & $49,093,615$ & $41,518,476$ & $36,335,584$ & $17,609,114$ & $46,197,106$ & $46,295,394$ \\
\hline 0.005 & 200 & $24,353,694$ & $61,186,311$ & $57,729,473$ & $49,975,077$ & $22,229,640$ & $54,411,136$ & $54,471,278$ \\
\hline 0.003 & 333 & $26,701,702$ & $70,352,522$ & $72,454,653$ & $62,701,669$ & $26,379,254$ & $60,324,047$ & $60,502,133$ \\
\hline 0.001 & 1000 & $31,751,468$ & $90,622,841$ & $113,912,076$ & $100,523,240$ & $38,087,792$ & $72,648,545$ & $72,411,820$ \\
\hline
\end{tabular}


Table 4.9 Per Aggregate TVaR (in 000s)

\begin{tabular}{|c|c|c|c|c|c|c|c|c|}
\hline EP & $\mathrm{T}$ & exp & gamma & Inorm & Pareto & loglogistic & GP \\
\hline 0.1 & 10 & $15,180,319$ & $28,706,210$ & $23,710,398$ & $21,839,121$ & $12,129,685$ & $29,906,938$ & $29,920,672$ \\
\hline 0.05 & 20 & $18,366,367$ & $39,451,462$ & $34,301,080$ & $30,922,402$ & $15,393,515$ & $38,555,438$ & $38,554,621$ \\
\hline 0.03 & 33 & $20,714,375$ & $47,869,927$ & $44,110,745$ & $39,397,732$ & $18,304,676$ & $44,781,103$ & $44,783,624$ \\
\hline 0.025 & 40 & $21,552,415$ & $50,955,705$ & $48,080,648$ & $42,860,541$ & $19,466,234$ & $46,973,276$ & $46,998,387$ \\
\hline 0.01 & 100 & $25,764,141$ & $66,973,996$ & $72,361,148$ & $64,585,175$ & $26,482,970$ & $57,757,487$ & $57,724,831$ \\
\hline 0.005 & 200 & $28,950,190$ & $79,537,701$ & $96,358,362$ & $87,103,463$ & $33,398,130$ & $65,663,195$ & $65,617,208$ \\
\hline 0.003 & 333 & $31,298,197$ & $88,986,141$ & $117,760,152$ & $108,114,586$ & $39,616,591$ & $71,354,158$ & $70,994,528$ \\
\hline 0.001 & 1000 & $36,347,964$ & $109,732,831$ & $176,731,007$ & $170,556,574$ & $57,177,474$ & $83,216,043$ & $82,869,083$ \\
\hline
\end{tabular}

The GPD performed the best for both per agg and per occ data for both VaR and TVaR estimation. Thus POT, focusing on tail structure, proves to be the most efficient method among other heavy-tailed distributions to estimate the tail-based risk measures.

\subsection{Losses Generated from Historical Landfalls}

The historical dataset contains annual losses generated from historical landfalls between 1900 and 2007 in Florida. The meteorological component uses the actual tracks derived from Hurricane Database (HURDAT) in National Hurricane Center (NHC) that contains hurricanes occurred in the state of Florida during that period.

The historical dataset consists of 108 data points, of which 50 are zeros. This sample size makes the empirical estimation of quantiles above $99 \%(107 / 108=0.9907)$ impossible.

FPHLM is developed to extrapolate losses in a scientific manner to solve such a problem. The distribution from historical landfalls is right skewed with a cluster of losses near 20 billion as it can be seen in Figure 4.11. In fitting GPD, thresholds between 5 billion and 7 billion have relatively stable shape parameters with values around -0.5 by MLE. In general, there are fewer losses above the selected thresholds. 


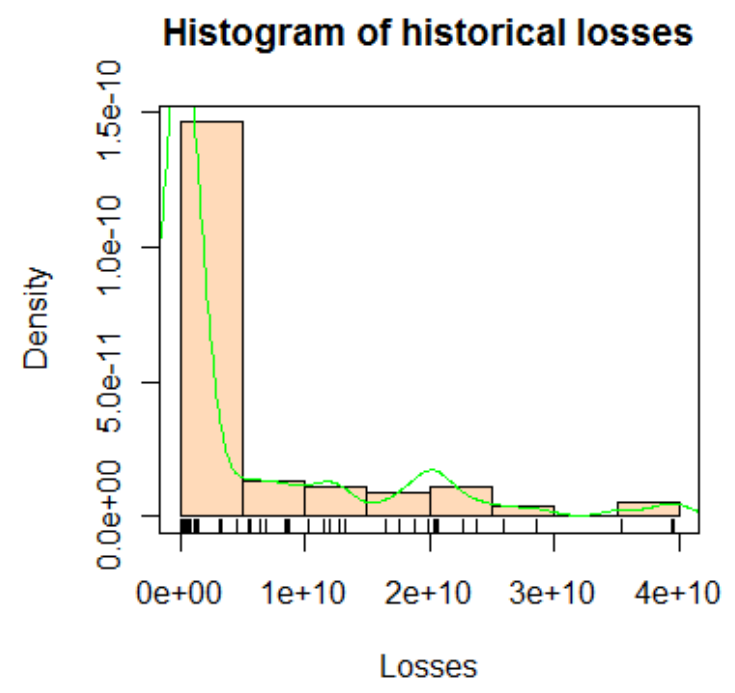

Figure 4.11 Histogram of losses generated by historical land falling and by-passing storms The simulated annual losses (per aggregate data) have mean relatively closer to the historical average while including 949 losses larger than the historical maximum to incorporate future uncertainty unrepresented in the data. The stochastic loss data was simulated for 54,000 years and produced 31,927 zero losses. This offsets the impact of data points populating the extremes on the mean. Extreme losses in the data set are responsible for the high kurtosis.

Table 4.10 Descriptive statistics for historical data and per agg data

\begin{tabular}{|c|c|c|c|c|c|}
\hline data & mean & std & skewness & kurtosis & Max \\
\hline Hist & $4,948,355,340$ & $9,161,389,501$ & 2.060 & 6.780 & $39,506,704,205$ \\
\hline Agg & $4,596,495,840$ & $9,996,759,996$ & 3.076 & 15.047 & $139,359,000,000$ \\
\hline
\end{tabular}


Table 4.11 Historical VaR (in 000s)

\begin{tabular}{|c|c|c|c|c|c|c|c|c|}
\hline $\mathrm{T}$ & Exp & Gamma & Lnorm & Pareto & Loglogis & GP & Historical & Modeled \\
\hline 10 & $14,642,038$ & $14,642,038$ & $11,227,150$ & $11,372,412$ & $9,047,777$ & $19,531,175$ & $20,360,744$ & $17,358,318$ \\
\hline 20 & $22,838,979$ & $22,838,979$ & $17,487,487$ & $17,047,239$ & $12,240,692$ & $26,831,013$ & $24,937,237$ & $26,270,450$ \\
\hline 33 & $29,342,899$ & $29,342,899$ & $23,319,310$ & $22,217,176$ & $15,177,607$ & $31,010,150$ & $33,553,918$ & $32,560,173$ \\
\hline 40 & $31,737,124$ & $31,737,124$ & $25,683,600$ & $24,300,879$ & $16,373,260$ & $32,298,970$ & $36,485,015$ & $34,996,373$ \\
\hline 100 & $44,216,497$ & $44,216,497$ & $40,155,622$ & $37,075,279$ & $23,866,194$ & $37,480,676$ & $39,495,880$ & $46,295,394$ \\
\hline
\end{tabular}

Table 4.12 Historical TVaR (in 000s)

\begin{tabular}{|c|c|c|c|c|c|c|c|c|}
\hline $\mathrm{T}$ & Exp & Gamma & Lnorm & Pareto & Loglogis & GP & Historical & Modeled \\
\hline 10 & $16,342,365$ & $15,897,407$ & $23,523,225$ & $22,556,224$ & $15,610,488$ & $28,089,609$ & $27,674,752$ & $29,920,672$ \\
\hline 20 & $19,772,303$ & $23,506,607$ & $33,192,929$ & $31,342,539$ & $20,825,032$ & $33,241,723$ & $33,761,441$ & $38,554,621$ \\
\hline 33 & $22,300,050$ & $29,756,424$ & $41,946,151$ & $39,347,137$ & $25,683,428$ & $36,191,294$ & $38,092,539$ & $44,783,624$ \\
\hline 40 & $23,202,242$ & $32,085,004$ & $45,444,881$ & $42,573,327$ & $27,670,066$ & $37,100,923$ & $39,446,570$ & $46,998,387$ \\
\hline 100 & $27,736,374$ & $44,360,921$ & $66,427,521$ & $62,351,888$ & $40,173,543$ & $40,758,092$ & $39,506,704$ & $57,724,831$ \\
\hline
\end{tabular}

The GPD estimated the historical PML better than other distributions as it is shown in

Table 4.11 and 4.12. The modeled losses tend to overestimate risk measures for including additional speculative losses.

The QQ plot for using GPD $(\mathrm{u}=6,000,000,000)$ in Figure 4.12 is approximately linear, implying a reasonable fit. At higher quantiles close to the 100 year PML, GPD tends to underestimate the impact. The return level plot gives a way to estimate the expected return level, the magnitude of losses that is expected to be exceeded on an average for a given return period. The points in the plot are empirically estimated return levels from the losses generated from historical landfalls. For example, one would expect annual losses in Florida from a hurricane to exceed $\$ 39,506,704,205$ (the maximum in historical data set) on an average of every 80 years. The curve produced by GPD is expected to 
asymptotically level off at the upper bound of $u-\frac{\sigma}{\xi}$ because of the negative shape parameter.
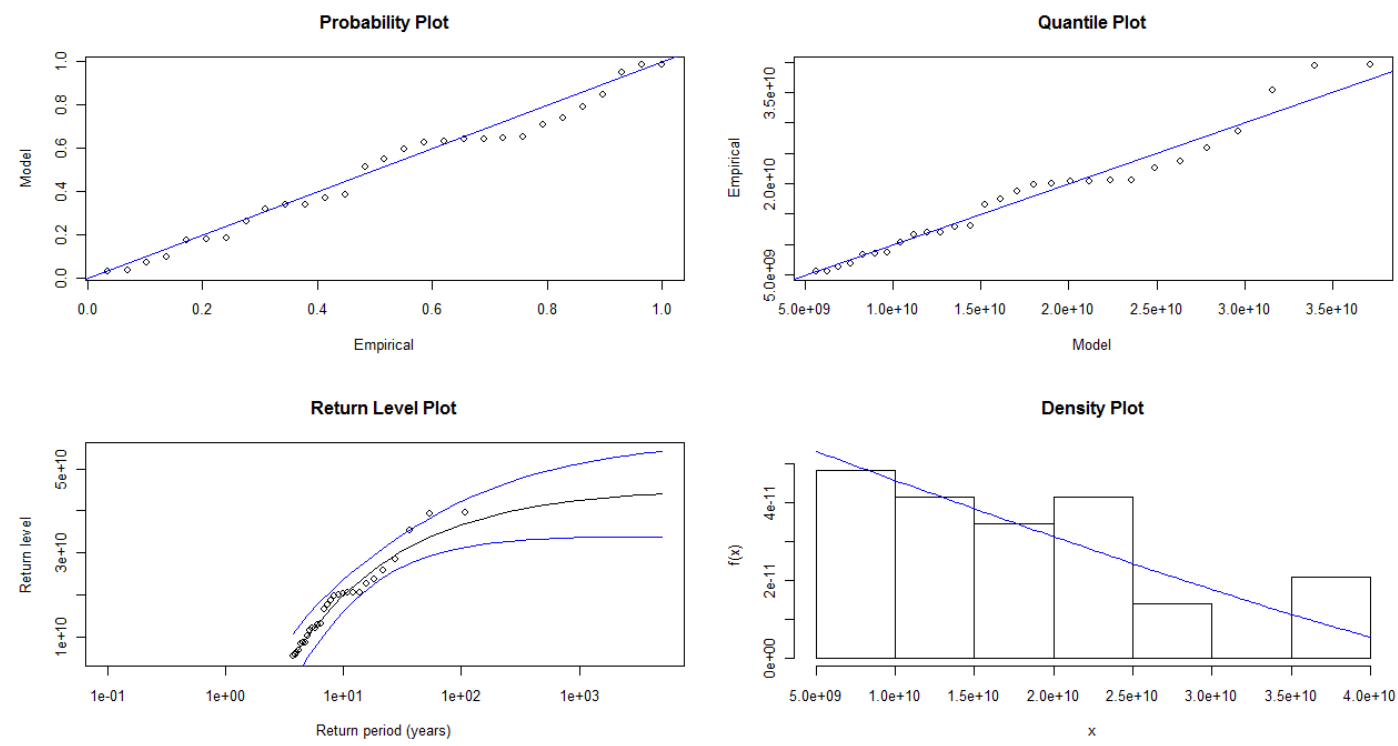

Figure 4.12 Diagnostic plots for GPD modeling historical losses

Simple model validation can be done by QQ plot. The linear trend in Figure 4.13 indicates that the model reasonably captures the characteristics of historical losses.

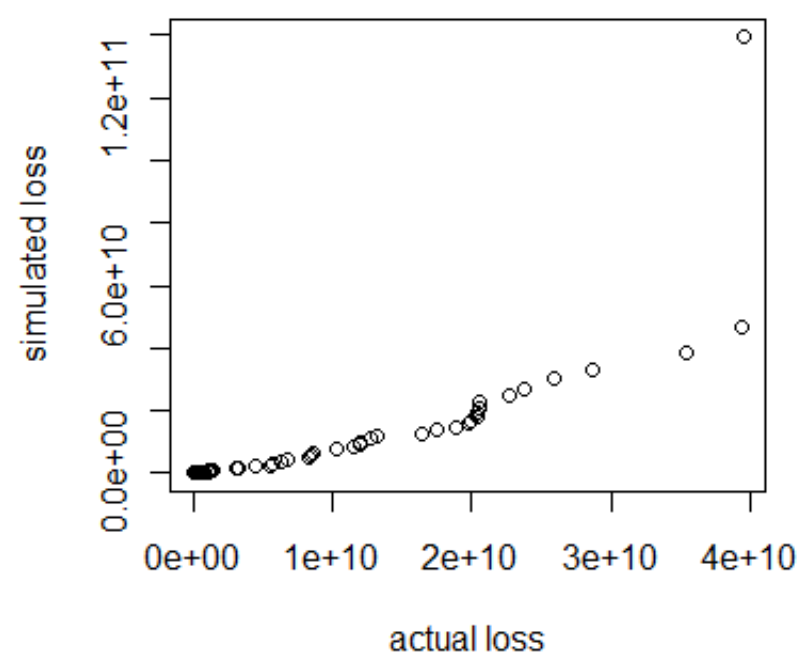

Figure 4.13 QQ plot for historical losses and stochastic losses (per agg) 


\section{Chapter 5}

\section{DISCUSSION ON MODEL LIMITATIONS}

The purpose of this research was to estimate the annual PML from FPHLM data. The accuracy of the results depends highly on the loss-generating model and good understanding of assumptions in every component of FPHLM is critical. My study attempted to extract loss distributions of simulated wind storm events to calculate risk measures. The smaller the exceedance probability, the larger the sample size needed to make inferences. The benefit of using cat models such as FPHLM lies in their ability to produce a large loss dataset that mimics the historical losses to make extreme quantile estimation possible.

In reality, the small sample size of extreme data is insufficient to provide an empirical basis for extrapolation beyond the available data and this leads to the extreme value paradigm to base tail structures on asymptotic models. As in any kind of parametric method, modeling risks exist due to the discrepancy between the model and reality. EVT has been proven to be a potentially good tool for modeling rare phenomena as it can be seen in Chapter 4 using GPD. However, full understanding of the characteristics of the loss data and awareness of model limitations become more crucial in extreme loss analysis as we substitute real data with theories (Dutta and Perry, 2006).

In historical data, the risk analysis completely depends on the historical incidents and an unverifiable assumption that the past is representative of the future. As McNeil (1997) points out "Extreme value methods based on rigorous mathematical theory do not predict the future with certainty, but they do offer good models for explaining the extreme events we have seen in the past", inferring future from the properties of the past sample requires 
caution. Possibility of losses that are not in the sample of the past realizations needs to be considered. The loss model constructed at IHRC seemed to follow the traits of the historical data while including speculation on the unknown future through additional extreme losses. The improvement of hurricane model is necessary and made possible by incorporating the most recent scientific knowledge on natural hazards in the meteorology component and advancing the understanding of secondary uncertainty in engineering component. Accordingly, statistics plays a significant role in such an endeavor.

The nature of extreme events is featured by its diminishing probabilities attaching to rapidly increasing damages. It is impossible to incorporate all the low occurrence highimpact events into a model, making overreliance on any modeled estimates vulnerable to "Black Swan" (Taleb, 2010). A Black Swan is an extreme event that is hard to predict but has significant consequences. It creates instability in averages and can ruin prediction. One of the main purposes of statistics is to provide a basis for decision making and strategic planning. Whatever model is selected, the uncertainty in the estimates needs to be understood and possibly measured in terms of confidence intervals and error rates. Since a model is an approximation of the reality and can cause costly estimation errors, a critical attitude towards modeling is required when translating information of data properties into decision making. The interaction of mathematics of randomness and human decision process needs to be further explored.

An evaluation of the estimated risk measures in this study was conducted. Table 5.1 suggests variability inherent in the stochastic data and Table 5.2 reveals variability of the parameter estimates. The extreme quantile estimation is generally volatile. The more extreme it gets, the wider the confidence interval width grows, reflecting that there is 
much less information available in the data about the behavior of the high return level compared with lower levels where data are relatively abundant. At the 20 year return period, the width exceeds $\$ 1$ billion as shown in Table 5.1. Thus as a generalized best guess, one single PML cannot provide an adequate representation of loss exposure due to large confidence interval width.

Table 5.1 Empirical PML estimation with $95 \%$ confidence bonds and interval width (in 000s)

\begin{tabular}{|c|c|c|c|c|}
\hline $\mathrm{T}$ & Lower & PML & Upper & Width \\
\hline 10 & $16,975,760$ & $17,358,318$ & $17,708,673$ & 732,914 \\
\hline 20 & $25,672,326$ & $26,270,450$ & $26,797,422$ & $1,125,095$ \\
\hline 33 & $31,972,117$ & $32,560,173$ & $33,215,071$ & $1,242,954$ \\
\hline 40 & $34,296,239$ & $34,996,373$ & $35,669,216$ & $1,372,977$ \\
\hline 100 & $45,236,194$ & $46,295,394$ & $47,254,760$ & $2,018,566$ \\
\hline 200 & $53,235,023$ & $54,471,278$ & $56,504,772$ & $3,269,749$ \\
\hline 333 & $59,032,322$ & $60,502,133$ & $62,330,823$ & $3,298,501$ \\
\hline 1000 & $68,924,089$ & $72,411,820$ & $74,245,942$ & $5,321,854$ \\
\hline
\end{tabular}

Table 5.2 Risk measure comparison for per aggregate data using GPD $(u=20,000,000,000)$

\begin{tabular}{|c|c|c|c|c|}
\hline & \multicolumn{2}{|c|}{ PML } & \multicolumn{2}{c|}{ TVaR } \\
\hline $\mathrm{n} \backslash \mathrm{xi}$ & -0.05564 & -0.01688 & -0.05564 & -0.01688 \\
\hline 10 & $17,255,602$ & $17,266,728$ & $29,746,244$ & $30,128,679$ \\
\hline 20 & $26,221,181$ & $26,279,612$ & $38,239,271$ & $38,991,951$ \\
\hline 33 & $32,610,679$ & $32,854,628$ & $44,291,995$ & $45,457,823$ \\
\hline 40 & $34,847,569$ & $35,187,658$ & $46,410,985$ & $47,752,125$ \\
\hline 100 & $45,752,216$ & $46,804,626$ & $56,740,877$ & $59,176,254$ \\
\hline 200 & $53,639,697$ & $55,473,921$ & $64,212,630$ & $67,701,641$ \\
\hline 1000 & $59,260,868$ & $61,798,284$ & $69,537,523$ & $73,921,021$ \\
\hline 2000 & $70,822,155$ & $75,216,481$ & $80,489,445$ & $87,116,478$ \\
\hline
\end{tabular}

Even if the right model is chosen, parameter risks are not reducible. The $95 \%$ profilelikelihood confidence interval for the shape parameter of GPD modeling annual 
aggregate losses is $(-0.05564,-0.0168)$. The risk measures differ noticeably at higher return periods, highlighting the parameter risks. From Table 5.2, the VaR estimation can go up by as much as $6.2 \%$ ( $\$ 4.4$ billion) and TVaR by $8.2 \%$ (approximately $\$ 6.6$ billion). The sensitivity of PML over various shape parameters near zero would not cause material changes in quantile estimation. However, a small change in shape estimate (especially when $\xi \geq \frac{1}{2}$ ) could result in a dramatic difference in the estimation of risk measures. Because of this property, sensitivity of $\xi$, also referred to as extreme value index and Pareto index $\alpha$ in Chapter 3 can be used in designing stress tests to incorporate effect of the unseen (Taleb, 2007). By varying $\alpha$ in the power laws, possible effects of inflation, climate changes and the cost of building can be included.

In general, theories allow for the building of a certain model to transform raw data into decision-assisting information, but a model built upon these theories usually possesses hidden weakness in terms of robustness and accuracy. The fundamental challenges of uncertainty in the tail, small sample statistics or low probability ultimately cannot be overcome in the mathematical and statistical domain; rather help may be sought in the realms of philosophy. Theories and other scientific methods are not black swan resistant as they tend to inaccurately generalize and simplify the reality.

Statistics can help unveil the sample properties and expose areas where knowledge is deemed fragile, but because of the variability in the extreme risk measure, it may fall short of providing a credible decision basis. In the banking and insurance sectors, taking extreme risks into consideration regulatory capital requirement is usually set at the $99 \%$ or $99.5 \%$ return level. Since the regulatory control is based only on a single quantile, 
PML has been long criticized for failing to be a robust tool to quantify the risks. There is a prolonged heated discussion on what risk measure to base the operational and strategic decisions on in the industry. Besides quantification through cat models, a systematic and serious effort to identify and evaluate risks underrepresented in the data is equally important. Decision making on uncertainty needs to balance the quantitative aspects and qualitative aspects of risk management. The study of judgment errors and cognitive limitations in modeling and data interpretation may help efficient decision making. 


\section{Chapter 6}

\section{CONCLUSIONS}

Cat events are hard to predict and hence hard to protect against, yet their economic and social consequences are enormous, making the development of reliable prediction methods valuable. Risk measures quantify the losses. They help risk assessment and support decisions on risk mitigation and prevention in both private and public sectors.

Statistical methodologies used to get risk measures for catastrophe losses can be nonparametric and parametric. Parametric methods use curve fitting and calculate quantiles as a function of distribution parameters. This can be accomplished by distributions fitting the whole data range or the POT method to use a high threshold to divide a distribution into a body and a tail. Extreme value theory provides simple techniques for estimating the probability of extremes. POT may be the best parameter method available for this estimation problem. The results indicate that the hurricane loss data generated from FPHLM were not as heavy tailed as expected and an exponential distribution seemed to explain the characteristics of the tail in per occurrence data. PMLs estimated using GPD were generally robust in this study. Interpretation of modeled results needs broad-minded thinking due to the uncertainty in the estimates of risk measures. The limitations and uncertainties of the model should be taken fully into consideration before basing any strategies on its inference.

One critical assumption made in the study is that the losses are identically and independently distributed. However, in situations where one hurricane follows another and losses in certain regions correlate with each other, EVT cannot model such dependency sufficiently. This study solely focuses on univariate distributions. 
Practitioners who are interested in hurricane losses associated with flood losses or aggregated losses from different lines of business in a disaster may want to explore bivariate and multivariate models to address these issues. The studies may want to approach the dependence structure of risks through copula models. The suitable copula models for catastrophe risks still need to be identified. This thesis mentioned an advantage of using flexible general class distributions such as Transformed Beta. The follow-up research may focus on this distribution to accommodate a wider variety of underlying data. 


\section{REFERENCE}

Balkema, A.A. and de Hann, L. (1974). Residual life time at great age. Annual Probability, 2 792-804.

Beirlant, J., Goegebeur, Y., Segers, J. and Teugels, J. (2004). Statistics of extremes: theory and application. John Wiley \& Sons.

Bermudez, P. and Kotz, S. (2010). Parameter estimation of the generalized pareto distribution-part I and part II. Journal of Statistical Planning and Inference,140, 13531388.

Bernardara, P., Schertzer, D., Sauquet, E., Tchiguirinskaia, I., and Lang, M. (2008). The flood probability distribution tail: how heavy is it? Stoch Environ Res Risk Assess, 22:107-122.

Bingham, N. H., Goldie, C. M. and Teugels, J. L. (1987). Regular Variation. Cambridge University Press, Cambridge

Brodin, E. and Rootzen, H. (2009). Univariate and bivariate GPD methods for predicting extreme wind storm losses. Insurance: Mathematics and Economics ,44, 345-356.

Coles, S. (2001). Extreme value theory: an introduction. Springer.

Coles, S. and Pericchi, L. (2003). Anticipating catastrophes through extreme value modeling. Applied Statistics 52, Part 4, 405-416.

Degen, M. and Embrechts, P. (2007). EVT-based estimation of risk capital and convergence of high quantiles. Preprint, ETH Zurich.

Dupuis, J. (1998). Exceedances over high thresholds: a guide to threshold selection. Extremes, 1(3) 251-261.

Dutta, K. and Perry, J. (2006). A tale of tails: an empricial analysis of loss distribution models for estimating operational risk capital. Working paper, Federal Reserve Bank of Boston, No.06-13.

Embrechts, P., Klupperlberg, C. and Mikosch, T.(1997). Modeling extremal events for insurance and finance. Springer.

Fisher, R.A. and Tippett, L.H.C. (1928). Limiting forms of the frequency distribution of the largest and smallest member of a sample. Proc. Camb. Phil. Soc., 24, 180-190.

Gilli, M. and Evis, K. (2003). An application of extreme value theory for measuring risk, Elsevier Science. 
Gnedenko, B.V. (1943). Sur la distribution limite du terme maximum d'ine serie aleatories. Ann. Math., 44, 423-453.

Gumbel, E.J. (1958). Statistics of Extremes. Columbia University Press, New York

Hamid, S., Kibria, B.M.G, Gulati, S. et.al (2008). Public hurricane loss evaluation models: predicting losses of residential structures in the State of Florida. JSM 2008 proceedings, 1052-1059.

Hamid, S., Kibria, B.M.G., Gulati, S. et al. (2010). Predicting losses of residential structures in the State of Florida by the public hurricane loss evaluation models with discussion. Statistical Methodology, Vol. 7, 552-573.

Juarez, S. and Schucany, W. (2004). Robust and efficient estimation for the generalized Pareto distribution. Extremes 7, 237-251.

Klugman, S., Panjer, H. and Willmot, G. (2008) Loss Model: from data to decisions (3 ${ }^{\text {rd }}$ edition) John Wiley \& Sons, Inc

Kreps, R. (2000) Continuous distribution. Retrieved from http://ermbook.guycarp.com/portal/books/pdf/continuous_distributions.pdf.

Kotz, S. and Nadarajah, S. (2000). Extreme value distributions: theory and application. Imperial College Press.

McNeil, A. (1997). Estimating the tails of loss severity distributions using extreme value theory. Astin Bulletin. Vol. 27, No.1, 117-137.

McNeil, A., Frey, R., Embrechts, P. (2005). Quantitative risk management-concepts, techniques and tools. Princeton University Press.

Peng, L. and Welsh, A. (2002). Robust estimation of the generalized Pareto distribution. Extremes. 4(1), 53-65.

Pfeifer, D. (2000). Scientific consulting in reinsurance brokerage: models, experiences, developments. Zeitschrift fur Versicherungsweasen 21, 771-777.

Reiss, R. and Thomas, M. (2008). Statistical analysis of extreme values: from insurance, finance, hydrology and other fileds ( $3^{\text {rd }}$ edition,) Springer Science and Business Media.

Resnick, S. (2007). Heavy-tail phenomena: probabilistic and statistical modeling. Spring Series in Operations Research and Financial Engineering. 
Stehlik, M., Potocky, R., Waldl, H. and Fabian, Z. (2010). On the favorable estimation for fitting heavy tailed data. Computational Statistics 25:485-503.

Taleb, N. (2007). Black swans and the domains of statistics. The American Statistician, August 2007 Vol. 81. No. 3:198-200.

Taleb, N. (2010). The black swan - the impact of the highly improbable. $2^{\text {nd }}$ edition. Random House Trade Paperback.

Wang, H. and Tsai, C. (2009).Tail index regression. UC Davis Graduate School of Management Research Paper No. 08-09. Retrieved from http://ssrn.com/abstract=1340758. 


\section{APPENDIX}

\section{A. Tail properties}

A standard theory for describing heavy-tailed behavior of statistical models is Karamata's theory of regular variation; see Bingham et.al (1987) and Embrechts et.al (1997).

For a measureable function $\mathrm{L}: \square \rightarrow(0, \infty)$ is slowing varying $(L \in S V)$ if for $\mathrm{t}>0$ :

$\lim _{x \rightarrow \infty} \frac{L(t x)}{L(x)}=1$

Slowly varying functions are functions which, in comparison with power functions, change relatively slowly for large $\mathrm{x}$, an example being the logarithm $\mathrm{L}(\mathrm{x})=\operatorname{In}(\mathrm{x})$. Regularly varying functions (regularly varying at $\infty$ with index $\alpha \in \square\left(f \in R V_{\alpha}\right)$ ) are functions which can be represented by power functions multiplied by slowly varying functions: $f=x^{\alpha} L(x)$.

For heavy-tail analysis, we assume $p(X>x)=\bar{F}(x)=x^{-\alpha} L(x)$, that is $\bar{F}(x) \in R V_{-\alpha}$, and focus on the problem of estimating the index of regular variation $\alpha$, which is one of the primary parameters of rare events. 


\section{B. Distributional Relationships and Characteristics}

The following graphs are cited from Klugman et al. (2008).

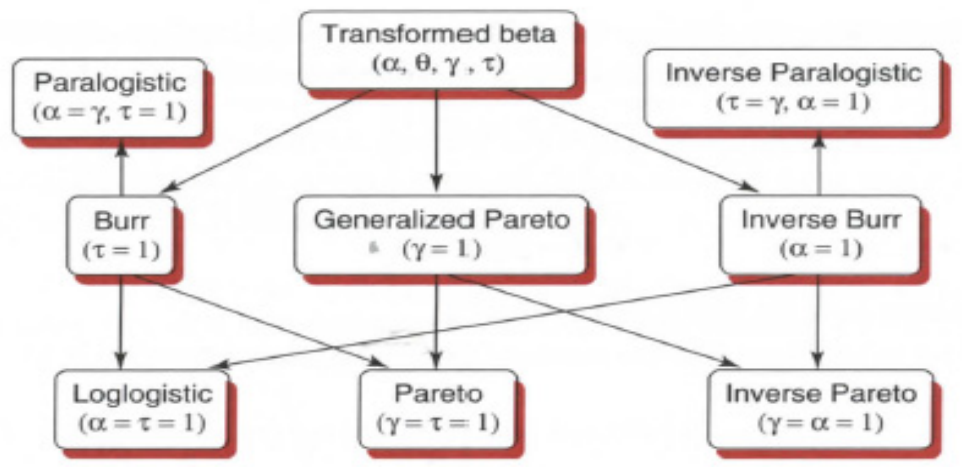

Figure B.1 Relationship between TB and others by setting specific values to parameters

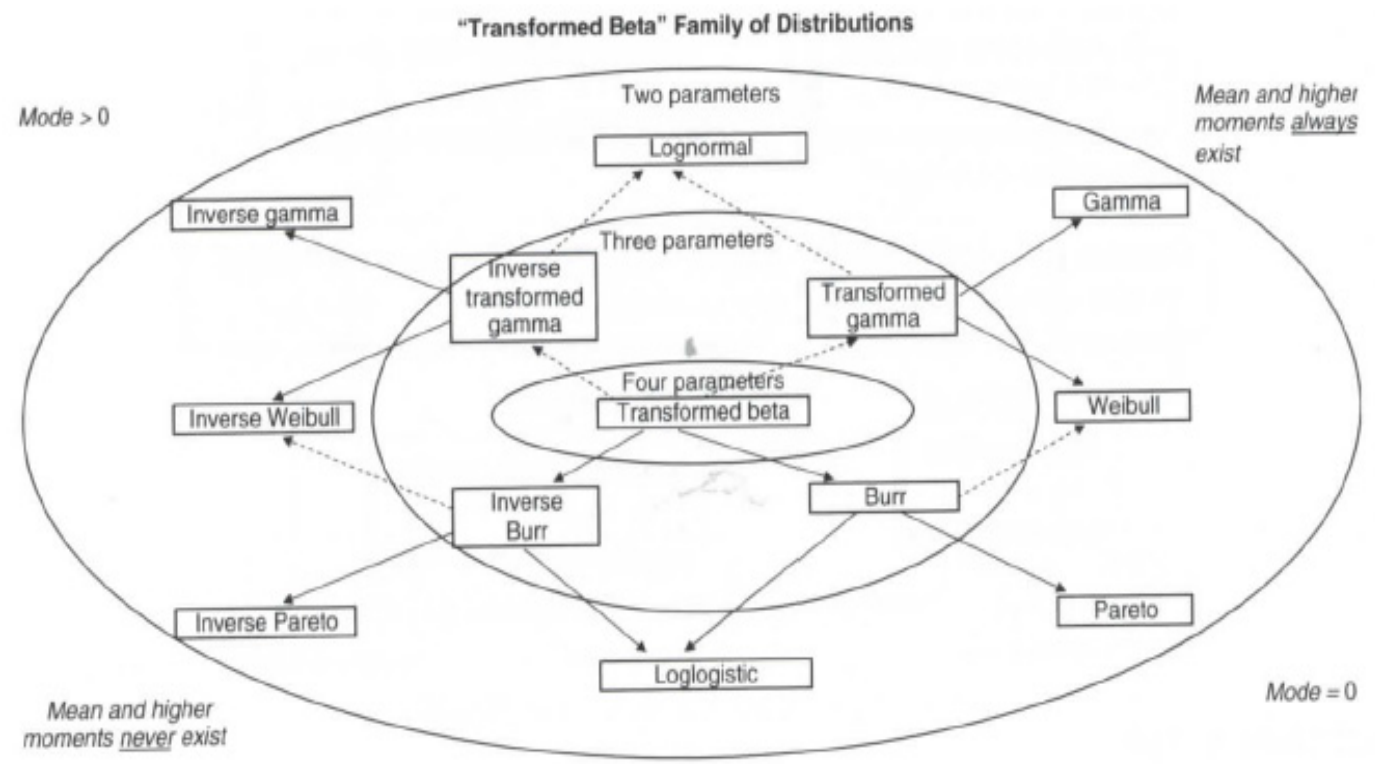

Figure B.2 Distributional relationships and characteristics

Straight line denotes special case and dot line represents limiting case. 


\section{Foundations of Peak-Over-Threshold Method}

C1. Maximum Domain of Attraction

Define $\left\{X_{1}, X_{2}, X_{3}, X_{4} \ldots\right\}$ as a sequence of i.i.d random variables from an unknown distribution $\mathrm{F}$ and $\mathrm{M}_{\mathrm{n}}=\max \left(\mathrm{X}_{1}, \ldots, \mathrm{X}_{\mathrm{n}}\right)$. The sequence of normalized maxima, $\frac{\left(M_{n}-b_{n}\right)}{a_{n}}$, converges in distribution:

$p\left(\frac{M_{n}-b_{n}}{a_{n}} \leq x\right)=F^{n}\left(a_{n} x+b_{n}\right) \rightarrow H(x), \mathrm{H}(\mathrm{x})$ is some non-degenerate df.

If this conditions satisfy, we call $\mathrm{F}$ is in the maximum domain of attraction of $\mathrm{H}$ and write as $F \in M D A(H)$. Fisher-Tippett (1928) theorem states that $\mathrm{H}(\mathrm{x})$ belongs to extreme value distribution family, denoting $H_{\xi}$.

For most applications it is sufficient to note that essentially all the common continuous distributions are in $\operatorname{MDA}\left(H_{\xi}\right)$ for some $\xi$.

C2. Balkema-de Haan-Pickands (1974) Theorem

If $F \in M D A(H)$,

$F^{[u]} \rightarrow W_{\xi, u, \sigma}(x)$ as $u \rightarrow \sup \{x: F(x)<1\}$

Where $F^{[u]}=p(X \leq x / X>u)=\frac{F(x)-F(u)}{1-F(u)}, x \geq u . W_{\xi, u, \sigma}(x)$ is GPD.

This theorem describes the limit distribution of excess loss over a sufficiently high threshold. This limiting result can be used as a general approximation to the true distribution of an exceedance without specifying the underlying distribution that generates the whole data. 


\section{C3. GPD Properties}

1. Let $\mathrm{Y}=\mathrm{X}-\mathrm{u} / \mathrm{X}>\mathrm{u}$ denote the conditional excess random variable. The conditional distribution of the excess over a threshold, $\mathrm{Z}=\mathrm{Y}-\mathrm{v} / \mathrm{Y}>\mathrm{v}$ is also GPD. Note $\mathrm{v}$ is an increase in threshold and the new threshold becomes $\mathrm{v}+\mathrm{u}$.

$$
\begin{aligned}
& p(Y \leq y)=W_{\xi, \sigma} \\
& p(Z \leq z)=p(Y \leq v+z / Y>v) \\
& =1-\frac{S_{Y}(v+z)}{S_{Y}(v)} \\
& =1-\left(\frac{1+\xi\left(\frac{v+z}{\sigma}\right)}{1+\xi\left(\frac{v}{\sigma}\right)}\right)^{-1 / \xi} \\
& =1-\left(\frac{\sigma+\xi(v+z)}{\sigma+\xi v}\right)^{-1 / \xi} \\
& =1-\left(1+\xi \frac{z}{\sigma+\xi v}\right)^{-1 / \xi} \\
& =W_{\xi, \sigma+\xi v}
\end{aligned}
$$

As shown above, excesses over a higher level $u+v$ also has a GP d.f. with the same shape $\xi$ and scale parameter $\sigma_{u+v}=\sigma_{u}+\xi v$. The property that shape parameter stays the same with increasing threshold describes the stability of GPD.

2. $E\left[X^{k}\right]=\infty$ for $k \geq 1 / \xi ;$ GPD has infinite mean if $\xi \geq 1$ 